\title{
The dressed nonrelativistic electron in a magnetic field
}

\author{
Laurent AMOUR, ${ }^{*}$ Benoît GRÉBERT ${ }^{\dagger}$ \\ and \\ Jean-Claude GUILLOT ${ }^{\ddagger}$
}

\begin{abstract}
We consider a nonrelativistic electron interacting with a classical magnetic field pointing along the $x_{3}$-axis and with a quantized electromagnetic field. When the interaction between the electron and photons is turned off, the electronic system is assumed to have a ground state of finite multiplicity. Because of the translation invariance along the $x_{3}$-axis, we consider the reduced Hamiltonian associated with the total momentum along the $x_{3}$-axis and, after introducing an ultraviolet cutoff and an infrared regularization, we prove that the reduced Hamiltonian has a ground state if the coupling constant and the total momentum along the $x_{3}$-axis are sufficiently small. We determine the absolutely continuous spectrum of the reduced Hamiltonian and, when the ground state is simple, we prove that the renormalized mass of the dressed electron is greater than or equal to its bare one. We then deduce that the anomalous magnetic moment of the dressed electron is non negative.
\end{abstract}

AMS classification numbers: 81V10, 81Q10, 81Q15

\footnotetext{
*Laboratoire de Mathématiques, UMR-CNRS 6056, Université de Reims, Moulin de la Housse - BP 1039, 51687 REIMS Cedex 2, France.

${ }^{\dagger}$ Laboratoire de Mathématiques Jean LERAY, UMR-CNRS 6629, Université de Nantes, 2, rue de la Houssinière, 44072 NANTES Cedex 03, France.

${ }^{\ddagger}$ Centre de Mathématiques Appliquées, UMR-CNRS 7641, Ecole Polytechnique, 91128 Palaiseau Cedex, France.
} 


\section{Contents}

1 Introduction $\quad 3$

2 Definition of the model and self-adjointness 5

2.1 The Pauli operator with magnetic fields . . . . . . . . . . 5

2.2 The model . . . . . . . . . . . . . . . . . . 6

2.3 Self-adjointness . . . . . . . . . . . . . . . . 7

2.4 The reduced Hamiltonian $\ldots \ldots \ldots \ldots$

3 Main results $\quad 12$

4 Proof of the main theorem $\quad 13$

4.1 Proof of (i) of theorem $4.1 \ldots \ldots \ldots \ldots \ldots$

4.2 Fundamental estimates . . . . . . . . . . . . . . . . 15

4.3 Proof of (iii) of theorem $4.1 \ldots \ldots \ldots \ldots$

A The Hamiltonian with infrared cutoff 22 


\section{Introduction}

We consider a nonrelativistic electron in $\mathbb{R}^{3}$ of charge $e$ and mass $m$ interacting with a magnetic field pointing along the $x_{3}$-axis and with photons. The magnetic field takes the form $\left(0,0, b\left(x_{1}, x_{2}\right)\right)$ with $b\left(x_{1}, x_{2}\right)=\frac{\partial a_{2}}{\partial x_{1}}\left(x_{1}, x_{2}\right)-\frac{\partial a_{1}}{\partial x_{2}}\left(x_{1}, x_{2}\right)$ where $a\left(x_{1}, x_{2}\right)$ is a vector potential. The associated Pauli Hamiltonian in Coulomb gauge is formally given by

$$
\begin{aligned}
H & =\frac{1}{2 m}\left(p-e a\left(x^{\prime}\right)-e A(x)\right)^{2}-\frac{e}{2 m} b\left(x^{\prime}\right) \sigma_{3} \otimes 1+V\left(x^{\prime}\right) \otimes 1 \\
& +1 \otimes H_{p h}-\frac{e}{2 m} \sigma \cdot B(x) .
\end{aligned}
$$

Here the units are such that $\hbar=c=1, p=-i \nabla_{x}, x=\left(x_{1}, x_{2}, x_{3}\right)$ together with $x^{\prime}=\left(x_{1}, x_{2}\right), \sigma=\left(\sigma_{1}, \sigma_{2}, \sigma_{3}\right)$ is the 3 -component vector of the Pauli matrices and $V\left(x^{\prime}\right)$ is an electric potential depending only on the transverse variables. The quantized electromagnetic field in the Coulomb gauge is formally given by

$$
\begin{gathered}
A(x)=\frac{1}{2 \pi} \sum_{\mu=1,2} \int d^{3} k\left(\frac{1}{|k|^{1 / 2}} \epsilon_{\mu}(k) e^{-i k \cdot x} a_{\mu}^{\star}(k)+\frac{1}{|k|^{1 / 2}} \epsilon_{\mu}(k) e^{i k \cdot x} a_{\mu}(k)\right) \\
B(x)=\frac{i}{2 \pi} \sum_{\mu=1,2} \int d^{3} k\left\{-|k|^{1 / 2}\left(\frac{k}{|k|} \wedge \epsilon_{\mu}(k)\right) e^{-i k \cdot x} a_{\mu}^{\star}(k)\right. \\
\left.+|k|^{1 / 2}\left(\frac{k}{|k|} \wedge \epsilon_{\mu}(k)\right) e^{i k \cdot x} a_{\mu}(k)\right\}
\end{gathered}
$$

where $\epsilon_{\mu}(k)$ are real polarization vectors satisfying $\epsilon_{\mu}(k) \cdot \epsilon_{\mu^{\prime}}(k)=\delta_{\mu \mu^{\prime}}, k$. $\epsilon_{\mu}(k)=0 ; a_{\mu}(k)$ and $a_{\mu}^{\star}(k)$ are the usual annihilation and creation operators acting in the Fock space

$$
\mathcal{F}:=\oplus_{n=0}^{\infty} L^{2}\left(\mathbb{R}^{3}, \mathbb{C}^{2}\right)^{\otimes_{s}^{n}}
$$

where $L^{2}\left(\mathbb{R}^{3}, \mathbb{C}^{2}\right)^{\otimes_{s}^{0}}=\mathbb{C}$ and $L^{2}\left(\mathbb{R}^{3}, \mathbb{C}^{2}\right)^{\otimes_{s}^{n}}$ is the symmetric $n$-tensor power of $L^{2}\left(\mathbb{R}^{3}, \mathbb{C}^{2}\right)$ appropriate for Bose-Einstein statistics. The annihilation and creation operators obey the canonical commutation relations $\left(a^{\sharp}=a^{\star}\right.$ or $\left.a\right)$

$$
\left[a_{\mu}^{\sharp}(k), a_{\mu^{\prime}}^{\sharp}\left(k^{\prime}\right)\right]=0 \quad \text { et } \quad\left[a_{\mu}(k), a_{\mu^{\prime}}^{\star}\left(k^{\prime}\right)\right]=\delta_{\mu \mu^{\prime}} \delta\left(k-k^{\prime}\right) .
$$

Finally the Hamiltonian for the photons is given by

$$
H_{p h}=\sum_{\mu=1,2} \int|k| a_{\mu}^{\star}(k) a_{\mu}(k) d^{3} k
$$

The Hilbert space associated with $H$ is then

$$
\mathcal{H}=L^{2}\left(\mathbb{R}^{3}, \mathbb{C}^{2}\right) \otimes \mathcal{F} \simeq L^{2}\left(\mathbb{R}^{3}, \mathbb{C}^{2} \otimes \mathcal{F}\right) .
$$


As it stands, the Hamiltonian $H$ cannot be defined as a self-adjoint operator in $\mathcal{H}$ and we need to introduce cutoff functions, both in $A(x)$ and in $B(x)$, which will satisfy appropriate hypothesis in order to get a self adjoint operator in $\mathcal{H}$.

This operator, still denoted by $H$, commutes with the third component, denoted by $P_{3}$, of the total momentum of the system (cf. [6]). We have $P_{3}=$ $p_{3} \otimes 1+1 \otimes \mathrm{d} \Gamma\left(k_{3}\right)$ where $\mathrm{d} \Gamma\left(k_{3}\right)$ is the second quantized operator associated to the multiplication operator by the third component of $k$ in $L^{2}\left(\mathbb{R}^{3}, \mathbb{C}^{2}\right)$. The spectrum of $P_{3}$ is the real line. In turns out that $H$ admits a decomposition over the spectrum of $P_{3}$ as a direct integral

$$
H \simeq \int_{\mathbb{R}}^{\oplus} H\left(P_{3}\right) d P_{3}
$$

on

$$
\mathcal{H} \simeq \int_{\mathbb{R}}^{\oplus} L^{2}\left(\mathbb{R}^{2}, \mathbb{C}^{2} \otimes \mathcal{F}\right) d P_{3} \simeq \int_{\mathbb{R}}^{\oplus} L^{2}\left(\mathbb{R}^{2}, \mathbb{C}^{2}\right) \otimes \mathcal{F} d P_{3} .
$$

The reduced operator $H\left(P_{3}\right)$ will be explicitely computed and the aim of this article is to initiate the spectral analysis of $H\left(P_{3}\right)$ when $\left|P_{3}\right|$ is small. The results of this work were announced in [1].

In the free case, i.e. when $b=V=0$, a similar problem has been studied in [12] by $\mathrm{T}$. Chen who considers a freely propagating nonrelativistic spinless charged particle interacting with the quantized electromagnetic field. Under an infrared regularization hypothesis, T. Chen proves that the reduced Hamiltonian associated to the total momentum $P$ has a unique ground state when $P$ is sufficiently small by applying the renormalization group method introduced in [9] (see also [30]). In the case of the one-particle sector of Nelson's model, a similar result has been obtained first by J. Fröhlich (see [19], [18]) and more recently by A. Pizzo (see [35], [36]) and J.S. Møller [34]. For a review of the mathematical problems of nonrelativistic quantum electrodynamics see [28].

The hypothesis about $b$ and $V$ (see section 2.1 for the precise assumption) are such that the electronic part of the Hamiltonian 1.1 has a finitely degenerated ground state and we face the problem of perturbing an eigenvalue of finite multiplicity at the bottom of an essential spectrum. In this paper we give a simple proof for the existence of a ground state for $H\left(P_{3}\right)$ with an ultraviolet cutoff and an infrared regularization. The proof borrows ideas both from [19] (see also $[18,35,36,20]$ ) where the Hamiltonian is invariant by translation and [10] (see also [11, 27, 25, 26, 29, 24, 32, 21] where the electronic part is confined).

When the ground state is simple we are able to prove that the renormalized mass of the electron is greater than or equal to its bare one and we deduce that the anomalous magnetic moment of the electron is non negative.

The question of removing the infrared regularization in such QED models for one electron is still open. Following [12], we can conjecture that, for $P_{3} \neq 0$, $H\left(P_{3}\right)$ without infrared regularization has no ground state in $\mathcal{H}$ (actually the ground state should leave the Fock space when the infrared regularization is turned off). 
The same proof also works for any free atom or positive ion interacting with the quantized electromagnetic field. Furthermore, in the neutral case, i.e. in the case of atoms, we can remove the infrared regularization by using a PowerZienau-Woolley transformation (see [2]).

Acknowledgements LA and BG acknowledge the hospitality of the Centre de Mathématiques Appliquées at the École Polytechnique where a large part of this work has been done.

\section{Definition of the model and self-adjointness}

The Hamiltonian $H$ can be written as

$$
H=H_{0}+H_{I}
$$

where

$$
H_{0}=\left\{\frac{1}{2 m} p_{3}^{2}+\frac{1}{2 m} \sum_{j=1,2}\left(p_{j}-e a_{j}\left(x^{\prime}\right)\right)^{2}-\frac{e}{2 m} b\left(x^{\prime}\right) \sigma_{3}+V\left(x^{\prime}\right)\right\} \otimes 1+1 \otimes H_{p h}(2
$$

and $H_{I}$ describes the interaction between the electron in the magnetic field $b\left(x^{\prime}\right)$

with the photons. A basic tool is now to describe the spectral properties of the Pauli operator in $L^{2}\left(\mathbb{R}^{2}, \mathbb{C}^{2}\right)$ that we are dealing with.

\subsection{The Pauli operator with magnetic fields}

Let $h(b, V)$ be the following operator in $L^{2}\left(\mathbb{R}^{2}, \mathbb{C}^{2}\right)$

$$
h(b, V)=\frac{1}{2 m} \sum_{j=1,2}\left(p_{j}-e a_{j}\left(x_{1}, x_{2}\right)\right)^{2}-\frac{e}{2 m} b\left(x_{1}, x_{2}\right) \sigma_{3}+V\left(x_{1}, x_{2}\right) .
$$

As in [31] the $a_{j}$ 's are real functions in $C^{1}\left(\mathbb{R}^{2}\right)$ such that

$$
b\left(x_{1}, x_{2}\right)=\frac{\partial}{\partial x_{1}} a_{2}\left(x_{1}, x_{2}\right)-\frac{\partial}{\partial x_{2}} a_{1}\left(x_{1}, x_{2}\right) .
$$

We suppose that $b$ and $V$ satisfy the following hypothesis:

Hyp. 2.1. $b$ and $V$ are such that $h(b, V)$ is essentially self adjoint on $C_{0}^{\infty}\left(\mathbb{R}^{2}, \mathbb{C}^{2}\right)$ and the bottom of the spectrum of $h(b, V)$ is a strictly negative isolated eigenvalue of finite multiplicity.

There exist many examples of $b$ and $V$ satisfying the hypothesis 2.1. Let us give one example.

Suppose that $b \in C^{1}\left(\mathbb{R}^{2}\right)$ and $V \in L^{\infty}\left(\mathbb{R}^{2}\right)$ sastisfy

$$
1 / C \leq b\left(x_{1}, x_{2}\right) \leq C \text { and }\left|\nabla b\left(x_{1}, x_{2}\right)\right| \leq C
$$


for some $C>1$ and

$$
V\left(x_{1}, x_{2}\right) \rightarrow 0 \text { as }\left|\left(x_{1}, x_{2}\right)\right| \rightarrow+\infty .
$$

We then have

Proposition 2.2. Suppose that $b$ and $V$ satisfy (2.4) and (2.5). Then the operator $h(b, V)$ with domain

$$
D(b, V)=\left\{u \in L^{2}\left(\mathbb{R}^{2}, \mathbb{C}^{2}\right) \mid h(b, V) u \in L^{2}\left(\mathbb{R}^{2}, \mathbb{C}^{2}\right)\right\}
$$

is self-adjoint in $L^{2}\left(\mathbb{R}^{2}, \mathbb{C}^{2}\right)$.

Furthermore $h(b, V)$ is essentially self-adjoint on $C_{0}^{\infty}\left(\mathbb{R}^{2}, \mathbb{C}^{2}\right)$.

The proof can be found in [7] (see also [17] and [22]).

According to [39] (see also $[15,40,37]) \frac{1}{2 m} \sum_{j=1,2}\left(p_{j}-e a_{j}\left(x_{1}, x_{2}\right)\right)^{2}-$ $\frac{e}{2 m} b\left(x_{1}, x_{2}\right) \sigma_{3}$ has zero as an eigenvalue of infinite multiplicity when $b$ satisfies (2.4). By adding $V\left(x_{1}, x_{2}\right)$ satisfying (2.5), the operator $h(b, V)$ may have eigenvalues of finite multiplicity accumulating at zero. In fact according to $[31,37]$ there exist $b$ and $V$ satisfying (2.4) and (2.5) such that hypothesis 2.1 is verified.

Notice that hypothesis (2.1) allows us to choose an uniform magnetic field but, in this case, $V$ cannot be identically zero.

\subsection{The model}

We now introduce the Hamiltonian in the Fock space associated to (1.1). As usual we will consider the charge $e$ in front of the quantized electromagnetic field $A(x)$ as a parameter that from now on we denote by $g$.

We introduce $\rho(k)$ a cutoff function associated with an ultraviolet cutoff and an infrared regularization. The precise assumption verified by $\rho$ will be given in each theorem but $\rho$ will always satisfy (2.9) below.

The associated quantized electromagnetic field is then given by $(j=1,2,3)$

$$
\begin{array}{r}
A_{j}(x, \rho)=\frac{1}{2 \pi} \sum_{\mu=1,2} \int d^{3} k\left(\frac{\rho(k)}{|k|^{1 / 2}} \epsilon_{\mu}(k)_{j} e^{-i k \cdot x} a_{\mu}^{\star}(k)\right. \\
\left.+\frac{\bar{\rho}(k)}{|k|^{1 / 2}} \epsilon_{\mu}(k)_{j} e^{i k \cdot x} a_{\mu}(k)\right), \\
B_{j}(x, \rho)=\frac{i}{2 \pi} \sum_{\mu=1,2} \int d^{3} k\left(-|k|^{1 / 2} \rho(k)\left(\frac{k}{|k|} \wedge \epsilon_{\mu}(k)\right)_{j} e^{-i k \cdot x} a_{\mu}^{\star}(k)\right. \\
\left.+|k|^{1 / 2} \bar{\rho}(k)\left(\frac{k}{|k|} \wedge \epsilon_{\mu}(k)\right)_{j} e^{i k \cdot x} a_{\mu}(k)\right) .
\end{array}
$$


The interaction Hamiltonian (cf. (2.1)) reads

$$
\begin{aligned}
H_{I}= & -\frac{g}{m} \sum_{j=1,2}\left\{A_{j}(x, \rho)\left(p_{j}-e a_{j}\left(x^{\prime}\right)\right)+\left(p_{j}-e a_{j}\left(x^{\prime}\right)\right) A_{j}(x, \rho)\right\} \\
& -\frac{g}{m}\left\{A_{3}(x, \rho) p_{3}+p_{3} A_{3}(x, \rho)\right\} \\
& -\frac{g}{2 m} \sigma \cdot B(x, \rho)+\frac{g^{2}}{2 m} A(x, \rho) \cdot A(x, \rho) .
\end{aligned}
$$

Noticing that $k \cdot \epsilon_{\mu}(k)=0, H_{I}$ can be rewritten as

$$
\begin{aligned}
H_{I}= & -\frac{g}{m} A_{3}(x, \rho) p_{3}-\frac{g}{m} \sum_{j=1,2} A_{j}(x, \rho)\left(p_{j}-e a_{j}\left(x^{\prime}\right)\right) \\
& -\frac{g}{2 m} \sigma \cdot B(x, \rho)+\frac{g^{2}}{2 m}: A(x, \rho) \cdot A(x, \rho):
\end{aligned}
$$

where we also substitute the Wick normal ordering : $A(x, \rho) \cdot A(x, \rho)$ : for $A(x, \rho)$. $A(x, \rho)$. This substitution changes the Hamiltonian by a constant as it follows from the canonical commutation relations.

Let $\mathcal{F}_{0, \text { fin }}$ be the set of $\left(\psi_{n}\right)_{n \geq 0} \in \mathcal{F}$ such that $\psi_{n}$ is in the Schwartz space for every $n$ and $\psi_{n}=0$ for all but finitely many $n$. Suppose that

$$
\int_{|k| \leq 1} \frac{|\rho(k)|^{2}}{|k|^{2}} d^{3} k<\infty \text { and } \int_{|k| \geq 1}|k||\rho(k)|^{2} d^{3} k<\infty .
$$

Then our model is described by the operator $H=H_{0}+H_{I}$ given by (2.2) and (2.8), and this operator is well defined on $C_{0}^{\infty}\left(\mathbb{R}^{3}, \mathbb{C}^{2}\right) \otimes \mathcal{F}_{0, \text { fin }}$.

\subsection{Self-adjointness}

In $L^{2}\left(\mathbb{R}^{3}, \mathbb{C}^{2}\right) \otimes \mathcal{F}$, the operator $H_{0}$ given by $(2.2)$ is essentially self adjoint on $C_{0}^{\infty}\left(\mathbb{R}^{3}, \mathbb{C}^{2}\right) \otimes \mathcal{F}_{0, \text { fin }}$ (see [38]). Its self-adjoint extension is still denoted by $H_{0}$. The interaction operator $H_{I}$ (see (2.8)) is a symmetric operator on $C_{0}^{\infty}\left(\mathbb{R}^{3}, \mathbb{C}^{2}\right) \otimes \mathcal{F}_{0, \text { fin }}$. We are going to prove that $H_{I}$ is relatively bounded with respect to $H_{0}$ to apply the Kato-Rellich theorem.

Theorem 2.3. Assume (2.9) and

$$
\frac{6|g|}{\pi \sqrt{2 m}}\left(\int \frac{|\rho(k)|^{2}}{|k|^{2}} d^{3} k\right)^{1 / 2}+\frac{g^{2}}{\pi^{2} m} \int \frac{|\rho(k)|^{2}}{|k|^{2}} d^{3} k<\frac{1}{2} .
$$

Then $H$ is a self-adjoint operator in $\mathcal{H}$ with domain $D(H)=D\left(H_{0}\right)$ and $H$ is essentially self-adjoint on $C_{0}^{\infty}\left(\mathbb{R}^{3}, \mathbb{C}^{2}\right) \otimes \mathcal{F}_{0, \text { fin }}$.

Proof. To begin with we recall the following well known estimates (cf. [8])

$$
\left\|a_{\mu}(g(., x)) \psi\right\| \leq\left(\int \frac{|g(x, k)|^{2}}{|k|} d^{3} k\right)^{1 / 2}\left\|\left(I \otimes H_{p h}^{1 / 2}\right) \psi\right\|
$$


and

$$
\begin{aligned}
\left\|a_{\mu}^{*}(g(., x)) \psi\right\| & \leq\left(\int \frac{|g(x, k)|^{2}}{|k|} d^{3} k\right)^{1 / 2}\left\|\left(I \otimes H_{p h}^{1 / 2}\right) \psi\right\| \\
& +\left(\int|g(x, k)|^{2} d^{3} k\right)^{1 / 2}\|\psi\| .
\end{aligned}
$$

We get for $\psi \in C_{0}^{\infty}\left(\mathbb{R}^{3}, \mathbb{C}^{2}\right) \otimes \mathcal{F}_{0, \text { fin }}$

$$
\begin{gathered}
\frac{|g|}{m}\left\|A_{3}(x, \rho) p_{3} \psi\right\| \leq \frac{4|g|}{\pi \sqrt{2 m}}\left(\int \frac{|\rho(k)|^{2}}{|k|^{2}} d^{3} k\right)^{1 / 2} \\
\left\|\left(I \otimes H_{p h}^{1 / 2}\right)\left(\frac{p_{3}}{\sqrt{2 m}} \otimes I\right) \psi\right\| \\
+\frac{2|g|}{\pi \sqrt{2 m}}\left(\int \frac{|\rho(k)|^{2}}{|k|} d^{3} k\right)^{1 / 2}\left\|\left(\frac{p_{3}}{\sqrt{2 m}} \otimes I\right) \psi\right\| .
\end{gathered}
$$

Denoting $e(b, V):=\inf \sigma(h(b, V))$, notice that

$$
\begin{aligned}
\left\|\left(I \otimes H_{p h}^{1 / 2}\right)\left(\frac{p_{3}}{\sqrt{2 m}} \otimes I\right) \psi\right\| & \leq \frac{1}{2}\left(\left\|\left(I \otimes H_{p h}\right) \psi\right\|+\left\|\left(\frac{p_{3}}{\sqrt{2 m}} \otimes I\right) \psi\right\|\right) \\
& \leq\left\|\left(H_{0}-e(b, V)\right) \psi\right\|
\end{aligned}
$$

and, for every $\epsilon>0$,

$$
\left\|\left(\frac{p_{3}}{\sqrt{2 m}} \otimes I\right) \psi\right\| \leq \sqrt{\frac{\epsilon}{2}}\left\|\left(H_{0}-e(b, V)\right) \psi\right\|+\frac{1}{\sqrt{2 \epsilon}}\|\psi\|
$$

to obtain that

$$
\begin{aligned}
\frac{|g|}{m}\left\|A_{3}(x, \rho) p_{3} \psi\right\| & \leq \frac{4|g|}{\pi \sqrt{2 m}}\left(\int \frac{|\rho(k)|^{2}}{|k|^{2}} d^{3} k\right)^{1 / 2}\left\|\left(H_{0}-e(b, V)\right) \psi\right\| \\
+\frac{2|g|}{\pi \sqrt{2 m}} & \left(\int \frac{|\rho(k)|^{2}}{|k|} d^{3} k\right)^{1 / 2}\left(\sqrt{\frac{\epsilon}{2}}\left\|\left(H_{0}-e(b, V)\right) \psi\right\|+\frac{1}{\sqrt{2 \epsilon}}\|\psi\|\right) .
\end{aligned}
$$

Therefore $\frac{g}{m} A_{3}(x, \rho) p_{3}$ is relatively bounded with respect to $H_{0}$ with relative bound $\frac{4|g|}{\pi \sqrt{2 m}}\left(\int \frac{|\rho(k)|^{2}}{|k|^{2}} d^{3} k\right)^{1 / 2}$. Similarly we verify that for $j=1,2, \frac{g}{m} A_{j}(x, \rho)\left(p_{j}-\right.$ $\left.e a_{j}\left(x^{\prime}\right)\right)$ is also relatively bounded with respect to $H_{0}$ with the same relative bound, and that $\frac{|g|}{2 m} \sigma \cdot B(x, \rho)$ is relatively bounded with respect to $H_{0}$ with a zero relative bound.

It remains to estimate the quadratic terms associated with $\frac{g^{2}}{2 m}: A(x, \rho)$. $A(x, \rho)$ :. Let us recall the following estimates (cf. [3]): 


$$
\begin{aligned}
\left\|a_{\mu}(f) a_{\lambda}(f) \psi\right\| & \leq\left(\int \frac{|\rho(k)|^{2}}{|k|^{2}} d^{3} k\right)\left\|\left(H_{p h}+1\right) \psi\right\| \\
& +K\left(\int \frac{|\rho(k)|^{2}}{|k|^{2}} d^{3} k\right)^{1 / 2}\left(\int|\rho(k)|^{2} d^{3} k\right)^{1 / 2}\left\|\left(H_{p h}+1\right)^{1 / 2} \psi\right\|, \\
\left\|a_{\mu}^{*}(f) a_{\lambda}(f) \psi\right\| \leq & \left(\int \frac{|\rho(k)|^{2}}{|k|^{2}} d^{3} k\right)\left\|\left(H_{p h}+1\right) \psi\right\| \\
& +\left(K\left(\int \frac{|\rho(k)|^{2}}{|k|^{2}} d^{3} k\right)^{1 / 2}\left(\int|\rho(k)|^{2} d^{3} k\right)^{1 / 2}\right. \\
& \left.+\left(\int \frac{|\rho(k)|^{2}}{|k|^{2}} d^{3} k\right)^{1 / 2}\left(\int \frac{|\rho(k)|^{2}}{|k|} d^{3} k\right)^{1 / 2}\right)\left\|\left(H_{p h}+1\right)^{1 / 2} \psi\right\|, \\
\left\|a_{\mu}^{*}(f) a_{\lambda}^{*}(f) \psi\right\| \leq & \left(\int \frac{|\rho(k)|^{2}}{|k|^{2}} d^{3} k\right)\left\|\left(H_{p h}+1\right) \psi\right\| \\
& +\left(K\left(\int \frac{|\rho(k)|^{2}}{|k|^{2}} d^{3} k\right)^{1 / 2}\left(\int|\rho(k)|^{2} d^{3} k\right)^{1 / 2}\right. \\
& \left.+\left(\int \frac{|\rho(k)|^{2}}{|k|^{2}} d^{3} k\right)^{1 / 2}\left(\int \frac{|\rho(k)|^{2}}{|k|} d^{3} k\right)^{1 / 2}\right)\left\|\left(H_{p h}+1\right)^{1 / 2} \psi\right\| \\
& +\left(\left(\int \frac{|\rho(k)|^{2}}{|k|^{2}} d^{3} k\right)^{1 / 2}\left(\int|k \| \rho(k)|^{2} d^{3} k\right)^{1 / 2}+\int \frac{|\rho(k)|^{2}}{|k|} d^{3} k\right)\|\psi\|
\end{aligned}
$$

where $K=\frac{1}{\pi} \int_{0}^{\infty} \frac{\sqrt{\lambda}}{(1+\lambda)^{2}}$.

As $\left(H_{p h}+1\right)^{1 / 2}$ is relatively bounded with respect to $H_{p h}+1$ (and thus to $\left.H_{0}\right)$ with a zero relative bound, we deduce that the relative bound of $\frac{g^{2}}{2 m}$ : $A(x, \rho) \cdot A(x, \rho)$ : with respect to $H_{0}$ is given by

$$
16 \frac{g^{2}}{2 m} \frac{1}{4 \pi^{2}} \int \frac{|\rho(k)|^{2}}{|k|^{2}} d^{3} k
$$

Finally we get that $H_{I}$ is relatively bounded with respect to $H_{0}$ with the relative bound

$$
\frac{12|g|}{\pi \sqrt{2 m}}\left(\int \frac{|\rho(k)|^{2}}{|k|^{2}} d^{3} k\right)^{1 / 2}+\frac{2 g^{2}}{\pi^{2} m} \int \frac{|\rho(k)|^{2}}{|k|^{2}} d^{3} k
$$

and hence theorem 2.3 is a consequence of the Kato-Rellich theorem.

\subsection{The reduced Hamiltonian}

The operator $H$ is invariant by translation in the $x_{3}$-direction. Thus, denoting by $P_{3}$ the total momentum in the $x_{3}$-direction $\left(P_{3}=p_{3} \otimes 1+1 \otimes \mathrm{d} \Gamma\left(k_{3}\right)\right), H$ 
has a direct integral representation in a spectral representation of $P_{3}$, i.e.

$$
H \simeq \int_{\mathbb{R}}^{\oplus} H\left(P_{3}\right) d P_{3}
$$

To compute $H\left(P_{3}\right)$ we proceed as in [19] (see also [6, 4] and[21]). Let $\Pi$ be the unitary map from $\mathcal{H}$ to $L^{2}(\mathbb{R}) \otimes L^{2}\left(\mathbb{R}^{2}, \mathbb{C}^{2}\right) \otimes \mathcal{F}$ defined by

$$
(\Pi \phi)_{n}\left(P_{3}, x^{\prime}, k_{1}, \ldots, k_{n}\right)=\hat{\phi}_{n}\left(x^{\prime}, P_{3}-\sum_{i=1}^{n} k_{i, 3}, x^{\prime}, k_{1}, \ldots, k_{n}\right)
$$

where the hat stands for the partial Fourier transform in $x_{3}$. One easily verifies that, on $C_{0}^{\infty}\left(\mathbb{R}^{3}, \mathbb{C}^{2}\right) \otimes \mathcal{F}_{0, \text { fin }}$

$$
\Pi A_{j}\left(x^{\prime}, x_{3}, \rho\right) \Pi^{*}=A_{j}\left(x^{\prime}, 0, \rho\right) .
$$

Therefore, for $\psi \in C_{0}^{\infty}\left(\mathbb{R}^{3}, \mathbb{C}^{2}\right) \otimes \mathcal{F}_{0, \text { fin }}$,

$$
\left(\Pi H \Pi^{*} \psi\right)\left(P_{3}, \cdot\right)=H\left(P_{3}\right) \psi\left(P_{3}, \cdot\right)
$$

where the reduced Hamiltonian $H\left(P_{3}\right)$ is given by

$$
H\left(P_{3}\right)=H_{0}\left(P_{3}\right)+H_{I}\left(P_{3}\right)
$$

with

$$
H_{0}\left(P_{3}\right)=h(b, V) \otimes 1+1 \otimes\left\{\frac{1}{2 m}\left(P_{3}-\mathrm{d} \Gamma\left(k_{3}\right)\right)^{2}+H_{p h}\right\}
$$

and

$$
\begin{array}{r}
H_{I}\left(P_{3}\right)=-\frac{g}{2 m} \sigma \cdot B\left(x^{\prime}, 0, \rho\right)-\frac{g}{m} \sum_{j=1,2} A_{j}\left(x^{\prime}, 0, \rho\right)\left(p_{j}-e a_{j}\left(x^{\prime}\right)\right) \\
-\frac{g}{m} A_{3}\left(x^{\prime}, 0, \rho\right)\left(P_{3}-\mathrm{d} \Gamma\left(k_{3}\right)\right)+\frac{g^{2}}{2 m}: A\left(x^{\prime}, 0, \rho\right) \cdot A\left(x^{\prime}, 0, \rho\right):
\end{array}
$$

For every $P_{3}, H\left(P_{3}\right)$ is now an operator in $L^{2}\left(\mathbb{R}^{2}, \mathbb{C}^{2}\right) \otimes \mathcal{F}$. We want to show that this formal operator defines a self-adjoint one such that (2.13) is satisfied.

The operator $\frac{1}{2 m}\left(P_{3}-\mathrm{d} \Gamma\left(k_{3}\right)\right)^{2}+H_{p h}$ is essentially self-adjoint on $\mathcal{F}_{0, f i n}$. Therefore, for every $P_{3} \in \mathbb{R}, H_{0}\left(P_{3}\right)$ is essentially self-adjoint in $C_{0}^{\infty}\left(\mathbb{R}^{2}, \mathbb{C}^{2}\right) \otimes$ $\mathcal{F}_{0, \text { fin }}$. We still denote by $H_{0}\left(P_{3}\right)$ its self-adjoint extension. On the other hand $H_{I}\left(P_{3}\right)$ is a symmetric operator on $C_{0}^{\infty}\left(\mathbb{R}^{2}, \mathbb{C}^{2}\right) \otimes \mathcal{F}_{0, \text { fin }}$ and we want to prove that it is relatively bounded with respect to $H_{0}\left(P_{3}\right)$. We then follow closely the lines of section 2.3 and we only focus on the estimates of the new terms. For $\psi \in C_{0}^{\infty}\left(\mathbb{R}^{2}, \mathbb{C}^{2}\right) \otimes \mathcal{F}_{0, \text { fin }}$ we have

$$
\begin{gathered}
\frac{|g|}{m}\left\|A_{3}\left(x^{\prime}, 0, \rho\right)\left(P_{3}-d \Gamma\left(k_{3}\right)\right) \psi\right\| \leq \frac{4|g|}{\pi \sqrt{2 m}}\left(\int \frac{|\rho(k)|^{2}}{|k|^{2}} d^{3} k\right)^{1 / 2} \\
\left\|\left(I \otimes H_{p h}^{1 / 2}\right)\left(I \otimes \frac{P_{3}-d \Gamma\left(k_{3}\right)}{\sqrt{2 m}}\right) \psi\right\| \\
+\frac{2|g|}{\pi \sqrt{2 m}}\left(\int \frac{|\rho(k)|^{2}}{|k|} d^{3} k\right)^{1 / 2}\left\|\left(I \otimes \frac{P_{3}-d \Gamma\left(k_{3}\right)}{\sqrt{2 m}}\right) \psi\right\| .
\end{gathered}
$$


For every component $\psi_{n}$ of $\psi \in \mathcal{F}_{0, \text { fin }}$ associated with $n$ photons the operator $\left(I \otimes H_{p h}^{1 / 2}\right)\left(I \otimes \frac{P_{3}-d \Gamma\left(k_{3}\right)}{\sqrt{2 m}}\right)$ is the multiplication operator by the function $\left(\sum_{i=1}^{n} \omega\left(k_{i}\right)\right)^{1 / 2} \frac{P_{3}-\sum_{i=1}^{n} k_{i, 3}}{\sqrt{2 m}}$. We then get

$$
\begin{aligned}
\left\|\left(I \otimes H_{p h}^{1 / 2}\right)\left(I \otimes \frac{P_{3}-d \Gamma\left(k_{3}\right)}{\sqrt{2 m}}\right) \psi\right\| & \leq \frac{1}{\sqrt{2}}\left\|\left(I \otimes\left\{H_{p h}+\frac{1}{2 m}\left(P_{3}-d \Gamma\left(k_{3}\right)\right)^{2}\right\}\right) \psi\right\| \\
& \leq \frac{1}{\sqrt{2}}\left\|\left(H_{0}\left(P_{3}\right)-e(b, V)\right) \psi\right\|
\end{aligned}
$$

and, for any $\epsilon>0$,

$$
\left\|\left(I \otimes \frac{P_{3}-d \Gamma\left(k_{3}\right)}{\sqrt{2 m}}\right) \psi\right\| \leq \sqrt{\frac{\epsilon}{2}}\left\|\left(H_{0}\left(P_{3}\right)-e(b, V)\right) \psi\right\|+\frac{1}{\sqrt{2 \epsilon}}\|\psi\| .
$$

Therefore

$$
\begin{gathered}
\frac{|g|}{m}\left\|A_{3}\left(x^{\prime}, 0, \rho\right)\left(P_{3}-d \Gamma\left(k_{3}\right)\right) \psi\right\| \leq \frac{2|g|}{\pi \sqrt{m}}\left(\int \frac{|\rho(k)|^{2}}{|k|^{2}} d^{3} k\right)^{1 / 2}\left\|\left(H_{0}\left(P_{3}\right)-e(b, V)\right) \psi\right\| \\
+\sqrt{\frac{\epsilon}{m}} \frac{|g|}{\pi}\left(\int \frac{|\rho(k)|^{2}}{|k|} d^{3} k\right)^{1 / 2}\left\|\left(H_{0}\left(P_{3}\right)-e(b, V)\right) \psi\right\| \\
+\frac{1}{\sqrt{\epsilon m}} \frac{|g|}{\pi}\left(\int \frac{|\rho(k)|^{2}}{|k|} d^{3} k\right)^{1 / 2}\|\psi\| .
\end{gathered}
$$

Thus, as in section 2.3, we obtain that, for any $\eta>0$, there exists a finite constant $a_{\eta}$ such that

$$
\left\|H_{I}\left(P_{3}\right) \psi\right\| \leq|g|(b+\eta)\left\|H_{0}\left(P_{3}\right) \psi\right\|+|g| a_{\eta}\|\psi\|
$$

with

$$
b=\frac{12}{\pi \sqrt{2 m}}\left(\int \frac{|\rho(k)|^{2}}{|k|^{2}} d^{3} k\right)^{1 / 2}+\frac{2 g}{\pi^{2} m} \int \frac{|\rho(k)|^{2}}{|k|^{2}} d^{3} k .
$$

Therefore we have

Theorem 2.4. Assume (2.9) and

$$
\frac{6|g|}{\pi \sqrt{2 m}}\left(\int \frac{|\rho(k)|^{2}}{|k|^{2}} d^{3} k\right)^{1 / 2}+\frac{g^{2}}{\pi^{2} m} \int \frac{|\rho(k)|^{2}}{|k|^{2}} d^{3} k<\frac{1}{2} .
$$

Then, for every $P_{3} \in \mathbb{R}, H\left(P_{3}\right)$ is a self-adjoint operator in $L^{2}\left(\mathbb{R}^{2}, \mathbb{C}^{2}\right) \otimes \mathcal{F}$ with domain $D\left(H\left(P_{3}\right)\right)=D\left(H_{0}\left(P_{3}\right)\right)$ and $H\left(P_{3}\right)$ is essentially self-adjoint on $C_{0}^{\infty}\left(\mathbb{R}^{2}, \mathbb{C}^{2}\right) \otimes \mathcal{F}_{0, \text { fin }}$

Further we get

Corollary 2.5. We have

$$
\Pi H \Pi^{*}=\int_{\mathbb{R}}^{\oplus} H\left(P_{3}\right) d P_{3} .
$$

The proof of corollary 2.5 follows by mimicking [4]. 


\section{Main results}

For a bounded below self-adjoint operator $A$ with a ground state, $m(A)$ will denote the multiplicity of $\inf \sigma(A)$. Our main result is the following theorem which states that, for $P_{3}$ and $g$ sufficiently small, $H\left(P_{3}\right)$ has a ground state:

Theorem 3.1. Assume that the cutoff function satisfies (2.9), (2.18) and

$$
\int_{|k| \leq 1} \frac{|\rho(k)|^{2}}{|k|^{3}} d^{3} k<\infty
$$

Then there exist $P>0$ and $g_{0}>0$ such that for $\left|P_{3}\right| \leq P$ and $|g| \leq g_{0}, H\left(P_{3}\right)$ has a ground state. Furthermore $m\left(H\left(P_{3}\right)\right) \leq m(h(b, V))$. In particular, if $e(b, V)$ is a simple eigenvalue of $h(b, V)$, then $\inf \sigma\left(H\left(P_{3}\right)\right)$ is a simple eigenvalue too.

The proof of this theorem is given in the next section.

\section{Remark 3.2.}

Notice that the regularization condition (3.1) does not allow $\rho(k)=1$ near the origin. According to [12], one may conjecture that $H\left(P_{3}\right)$ has no ground state for $P_{3} \neq 0$ when this infrared condition is not satisfied.

The existence of a ground state has several consequences. The first one is the existence of asymptotic Fock representations for the CCR.

For $f \in L^{2}\left(\mathbb{R}^{3}, \mathbb{C}^{2}\right)$, we define on $D\left(H_{0}\left(P_{3}\right)\right)$ the operators

$$
a_{\mu, t}^{\sharp}(f):=e^{i t H\left(P_{3}\right)} e^{-i t H_{0}\left(P_{3}\right)} a_{\mu}^{\sharp}(f) e^{i t H_{0}\left(P_{3}\right)} e^{-i t H\left(P_{3}\right)} .
$$

Let $Q$ be a closed null set such that the polarization vectors $\epsilon_{\mu}(k)$ are $C^{\infty}$ on $\mathbb{R}^{3} \backslash Q$ for $\mu=1,2$. We have

Corollary 3.3. Suppose that the hypothesis of theorem 3.1 are satisfied. Then, for $f \in C_{0}^{\infty}\left(\mathbb{R}^{3} \backslash Q\right)$ and for every $\Psi \in D\left(H_{0}\left(P_{3}\right)\right)$ the strong limits of $a_{\mu, t}^{\sharp}(f)$ exist:

$$
\lim _{t \rightarrow \pm \infty} a_{\mu, t}^{\sharp}(f) \Psi=: a_{\mu, \pm}^{\sharp}(f) \Psi .
$$

The $a_{\mu, \pm}^{\sharp}$ 's satisfy the $C C R$ and, if $\Phi\left(P_{3}\right)$ is a ground state for $H\left(P_{3}\right)$, we have for $f \in C_{0}^{\infty}\left(\mathbb{R}^{3} \backslash Q\right)$ and $\mu=1,2$

$$
a_{\mu, \pm}(f) \Phi\left(P_{3}\right)=0 .
$$

We then deduce the following corollary

Corollary 3.4. Under the hypothesis of theorem 3.1, the absolutely continuous spectrum of $H\left(P_{3}\right)$ equals to $\left[\inf \sigma\left(H\left(P_{3}\right)\right),+\infty\right)$. 
The proofs of these two corollaries follow by mimicking [27, 29].

Our last application concerns the renormalized mass and magnetic moment of the electron.

From now on we assume that the ground state of $h(b, V)$ is simple in such a way that the ground state of $H\left(P_{3}\right)$ is also simple. To state our result we need the notations and results of theorem 4.1. Let $E_{\sigma}\left(P_{3}\right)$ be the ground energy of the hamiltonian with infrared cutoff $H_{\sigma}\left(P_{3}\right), E_{\sigma}\left(P_{3}\right)$ is a simple and an isolated eigenvalue of $H_{\sigma}\left(P_{3}\right)$ and therefore we deduce from the standard Kato-Rellich perturbation theory that $E_{\sigma}\left(P_{3}\right)$ is a regular function of $P_{3}$. We then define the renormalized mass of the dressed electron by

$$
m^{\star}:=\liminf _{\sigma \rightarrow 0} m_{\sigma}^{\star}
$$

where

$$
\left(m_{\sigma}^{\star}\right)^{-1}=\partial_{P_{3}}^{2} E_{\sigma}(0)
$$

Let $g_{\mathrm{el}}$ be the magnetic moment of the dressed electron. We then have

Corollary 3.5. Under the hypothesis of theorem 3.1 and assuming that $e(b, V)$ is a simple eigenvalue, we have

$$
m^{\star} \geq m
$$

i.e. the renormalized mass of the dressed electron in a magnetic field is larger than or equal to the bare mass of the electron. It then follows that $g_{\mathrm{el}} \geq 2$.

Proof. Since the ground state of $H_{\sigma}\left(P_{3}\right)$ is non degenarate, $E_{\sigma}\left(P_{3}\right)$ and $\Phi_{\sigma}\left(P_{3}\right)$ are smooth function of the parameter $P_{3}$ and we easily obtain by differentiating the relation $H_{\sigma}\left(P_{3}\right) \Phi_{\sigma}\left(P_{3}\right)=E_{\sigma}\left(P_{3}\right) \Phi_{\sigma}\left(P_{3}\right)$ the following formulas

$$
\partial_{P_{3}} E_{\sigma}\left(P_{3}\right)=\left\langle\Phi_{\sigma}\left(P_{3}\right),\left(\partial_{P_{3}} H_{\sigma}\left(P_{3}\right)\right) \Phi_{\sigma}\left(P_{3}\right)\right\rangle
$$

and

$$
\begin{aligned}
\partial_{P_{3}}^{2} E_{\sigma}\left(P_{3}\right) & =\left\langle\Phi_{\sigma}\left(P_{3}\right),\left(\partial_{P_{3}}^{2} H_{\sigma}\left(P_{3}\right)\right) \Phi_{\sigma}\left(P_{3}\right)\right\rangle \\
& -2\left\langle\partial_{P_{3}} \Phi_{\sigma}\left(P_{3}\right),\left(H_{\sigma}\left(P_{3}\right)-E_{\sigma}\left(P_{3}\right)\right) \partial_{P_{3}} \Phi_{\sigma}\left(P_{3}\right)\right\rangle .
\end{aligned}
$$

As $\partial_{P_{3}}^{2} H_{\sigma}\left(P_{3}\right)=1 / m$ and $H_{\sigma}\left(P_{3}\right)-E_{\sigma}\left(P_{3}\right) \geq 0$ we obtain $m_{\sigma}^{\star} \geq m$ for all $\sigma$ and thus $m^{\star} \geq m$.

The fact that $\mathbf{g}_{\mathrm{el}} \geq 2$ follows from $m^{\star} \geq m$ as in $[13,14,41]$.

\section{Proof of the main theorem}

To begin with we introduce an infrared regularized cutoff in the interaction Hamiltonian $H_{I}\left(P_{3}\right)$. Precisely, for $\sigma>0$, let $\rho_{\sigma}$ be a $C_{0}^{\infty}$ regularization of $\rho$ such that

(i) $\rho_{\sigma}(k)=0$ for $|k| \leq \sigma$ 
(ii) $\lim _{\sigma \rightarrow 0} \int \frac{\left|\rho_{\sigma}(k)-\rho(k)\right|^{2}}{|k|^{j}} d^{3} k=0$ for $j=1,2,-1$.

We define $H_{I, \sigma}\left(P_{3}\right)$ as the operator obtained from (2.16) by substituting $\rho_{\sigma}(k)$ for $\rho(k)$. We then introduce

$$
H_{\sigma}\left(P_{3}\right)=H_{0}\left(P_{3}\right)+H_{I, \sigma}\left(P_{3}\right)
$$

and we set $E_{\sigma}\left(P_{3}\right):=\inf \sigma\left(H_{\sigma}\left(P_{3}\right)\right)$. Theorem 3.1 is a simple consequence of the following result (see [8])

Theorem 4.1. There exist $g_{0}>0, \sigma_{0}>0$ and $P>0$ such that, for every $g$ satisfying $|g| \leq g_{0}$, for every $\sigma$ satisfying $0<\sigma<\sigma_{0}$ and for every $P_{3}$ satisfying $\left|P_{3}\right| \leq P$, the following properties hold:

(i) For every $\Psi \in D\left(H_{0}\left(P_{3}\right)\right)$ we have $H_{\sigma}\left(P_{3}\right) \Psi \rightarrow_{\sigma \rightarrow 0} H\left(P_{3}\right) \Psi$

(ii) $H_{\sigma}\left(P_{3}\right)$ has a normalized ground state $\Phi_{\sigma}\left(P_{3}\right)$.

(iii) Fix $\lambda \in(e(b, V), 0)$. We have

$$
\left\langle\Phi_{\sigma}\left(P_{3}\right), P_{(-\infty, \lambda]} \otimes P_{\Omega_{p h}} \Phi_{\sigma}\left(P_{3}\right)\right\rangle \geq 1-\delta_{g}(\lambda)
$$

where $\delta_{g}(\lambda)$ tends to zero when $g$ tends to zero and $\delta_{g}(\lambda)<1$ for $|g| \leq g_{0}$.

In the last item, $P_{(-\infty, \lambda]}$ is the spectral projection on $(-\infty, \lambda]$ associated to $h(b, V)$ and $P_{\Omega_{p h}}$ is the orthogonal projection on $\Omega_{p h}$, the vacuum state in $\mathcal{F}$.

Theorem 3.1 is easily deduced from theorem 4.1 as follows. Let $\Phi_{\sigma}\left(P_{3}\right)$ be as in theorem 4.1 (ii). Since $\left\|\Phi_{\sigma}\left(P_{3}\right)\right\|=1$, there exits a sequence $\left(\sigma_{k}\right)_{k \geq 1}$ converging to zero such that $\left(\Phi_{\sigma_{k}}\left(P_{3}\right)\right)_{k \geq 1}$ converges weakly to a state $\Phi\left(P_{3}\right)$. On the other hand, since $P_{(-\infty, \lambda]} \otimes P_{\Omega_{p h}}$ is finite rank for $\lambda \in(e(b, V), 0)$, it follows from (iii) that for $|g| \leq g_{0}$ and $\left|P_{3}\right| \leq P_{0}$,

$$
\left\langle\Phi\left(P_{3}\right), P_{(-\infty, \lambda]} \otimes P_{\Omega_{p h}} \Phi\left(P_{3}\right)\right\rangle \geq 1-\delta_{g}(\lambda)
$$

which implies $\Phi\left(P_{3}\right) \neq 0$. Then we deduce from (i) and from a well known result ([5] lemma 4.9) that $\Phi\left(P_{3}\right)$ is a ground state for $H\left(P_{3}\right)$.

The result concerning the multiplicity of the ground state is an easy consequence of corollary 3.4 in [29].

So it remains to prove theorem 4.1. The assertion (i) is easily verified in section 4.1 below. The second assertion is proved in the appendix. Actually the proof of (ii) is lenghty but straightforward since with the infrared cutoff we have a control of the photon's number in term of the energy. The real difficult part is the third one which allows to relax the infrared cutoff. The fundamental lemma in the proof of (iii) is lemma 4.3 which states that, for $g$ and $P_{3}$ small enough, the difference $E_{\sigma}\left(P_{3}-k_{3}\right)-E_{\sigma}\left(P_{3}\right)$ is minorized by $-\frac{3}{4}|k|$ uniformly with respect to $\sigma$. This estimate, proved in section 4.2, is essential to control the number of photons in a ground state of $H_{\sigma}\left(P_{3}\right)$ via a pull through formula (cf. section 4.3). 


\subsection{Proof of (i) of theorem 4.1}

Let $\tilde{\rho}_{\sigma}:=\rho-\rho_{\sigma}$. We have

$$
\begin{aligned}
H\left(P_{3}\right)- & H_{\sigma}\left(P_{3}\right)=H_{I}\left(P_{3}\right)-H_{I, \sigma}\left(P_{3}\right) \\
& =-\frac{g}{2 m} \sigma \cdot B\left(x^{\prime}, 0, \tilde{\rho}_{\sigma}\right)-\frac{g}{m} \sum_{j=1,2} A_{j}\left(x^{\prime}, 0, \tilde{\rho}_{\sigma}\right)\left(p_{j}-e a_{j}\left(x^{\prime}\right)\right) \\
& -\frac{g}{m} A_{3}\left(x^{\prime}, 0, \tilde{\rho}_{\sigma}\right)\left(P_{3}-\mathrm{d} \Gamma\left(k_{3}\right)\right)+\frac{g^{2}}{2 m}: A\left(x^{\prime}, 0, \tilde{\rho}_{\sigma}\right) \cdot A\left(x^{\prime}, 0, \rho\right): \\
& +\frac{g^{2}}{2 m}: A\left(x^{\prime}, 0, \rho_{\sigma}\right) \cdot A\left(x^{\prime}, 0, \tilde{\rho}_{\sigma}\right):
\end{aligned}
$$

Therefore, as by hypothesis $\lim _{\sigma \rightarrow 0} \int \frac{\left|\tilde{\rho}_{\sigma}(k)\right|^{2}}{|k|^{j}} d^{3} k=0$ for $j=-1,1,2$, we deduce from the estimates of sections 2.3, 2.4 and from the Lebesgue's theorem that for every $\Psi \in D\left(H_{0}\left(P_{3}\right)\right)$,

$$
\left(H\left(P_{3}\right)-H_{\sigma}\left(P_{3}\right)\right) \Psi \rightarrow_{\sigma \rightarrow 0} 0 .
$$

\subsection{Fundamental estimates}

In this section we give two lemmas which allow to control the function

$$
P_{3} \mapsto E_{\sigma}\left(P_{3}\right) \text {. }
$$

Let $g_{1}>0$ such that (2.18) is satisfied for $|g| \leq g_{1}$.

Lemma 4.2. There exist $\sigma_{0}>0$ and a finite constant $C>0$ which does not depend on $\sigma \in\left(0, \sigma_{0}\right]$ and $P_{3} \in \mathbb{R}$ such that

$$
e(b, V)-|g| C \leq E_{\sigma}\left(P_{3}\right) \leq e(b, V)+\frac{P_{3}^{2}}{2 m}
$$

for every $\sigma \in\left(0, \sigma_{0}\right], P_{3} \in \mathbb{R}$ and $|g| \leq g_{1}$.

Proof. Let $\phi(b, V)$ be the normalized ground state of $h(b, V)$. Since $\left\langle a_{\mu}(k) \Omega_{p h}, \Omega_{p h}\right\rangle=$ $\left\langle\Omega_{p h}, a_{\mu}^{*}(k) \Omega_{p h}\right\rangle=0$, we have

$$
\begin{aligned}
\left\langle H_{\sigma}\left(P_{3}\right) \phi(b, V) \otimes \Omega_{p h}, \phi(b, V) \otimes \Omega_{p h}\right\rangle & =\left\langle H_{0}\left(P_{3}\right) \phi(b, V) \otimes \Omega_{p h}, \phi(b, V) \otimes \Omega_{p h}\right\rangle \\
& =e(b, V)+\frac{P_{3}^{2}}{2 m}
\end{aligned}
$$

and thus

$$
\begin{aligned}
E_{\sigma}\left(P_{3}\right) & :=\inf \left\{\left\langle H_{\sigma}\left(P_{3}\right) \Phi, \Phi\right\rangle \mid \Phi \in D\left(H_{0}\left(P_{3}\right)\right),\|\Phi\|=1\right\} \\
& \leq e(b, V)+\frac{P_{3}^{2}}{2 m} .
\end{aligned}
$$


On the other hand, let $\tilde{H}_{\sigma}$ be the following operator in $L^{2}\left(\mathbb{R}^{2}, \mathbb{C}^{2}\right) \otimes \mathcal{F}$ :

$$
\tilde{H}_{\sigma}=\tilde{H}_{0}+\tilde{H}_{I, \sigma}
$$

with

$$
\tilde{H}_{0}=h(b, V) \otimes I+I \otimes H_{p h}
$$

and

$$
\begin{aligned}
\tilde{H}_{I, \sigma} & =-\frac{g}{2 m} \sigma \cdot B\left(x^{\prime}, 0, \rho_{\sigma}\right) \\
& -\frac{g}{2 m} \sum_{j=1,2}\left(A_{j}\left(x^{\prime}, 0, \rho_{\sigma}\right)\left(p_{j}-e a_{j}\left(x^{\prime}\right)\right)+\left(p_{j}-e a_{j}\left(x^{\prime}\right)\right) A_{j}\left(x^{\prime}, 0, \rho_{\sigma}\right)\right) \\
& +\frac{g^{2}}{2 m} \sum_{j=1,2}: A_{j}\left(x^{\prime}, 0, \rho_{\sigma}\right) \cdot A_{j}\left(x^{\prime}, 0, \rho_{\sigma}\right):
\end{aligned}
$$

As in section 2.4 one easily checks that, for $|g| \leq g_{1}, \tilde{H}_{\sigma}$ is a self-adjoint operator in $L^{2}\left(\mathbb{R}^{2}, \mathbb{C}^{2}\right) \otimes \mathcal{F}$ with domain $D\left(H_{0}\left(P_{3}\right)\right)$. Furthermore, on $D\left(H_{0}\left(P_{3}\right)\right)$

$$
H_{\sigma}\left(P_{3}\right)=\tilde{H}_{\sigma}+\frac{1}{2 m}\left(P_{3}-d \Gamma\left(k_{3}\right)-g A_{3}\left(x^{\prime}, 0, \rho_{\sigma}\right)\right)^{2}-C(g, \sigma)
$$

where, in order to take into account the Wick normal ordering,

$$
C(g, \sigma):=\frac{g^{2}}{2 m} \frac{1}{(2 \pi)^{2}} \int \frac{\left|\rho_{\sigma}\right|^{2}}{|k|}\left(\sum_{\mu=1,2} \epsilon_{\mu}(k)_{3}^{2}\right) d^{3} k .
$$

Hence,

$$
\inf \sigma\left(\tilde{H}_{\sigma}\right) \leq E_{\sigma}\left(P_{3}\right)+C(g, \sigma)
$$

for every $P_{3} \in \mathbb{R}$. Furthermore there exists $\sigma_{0}>0$ such that for $0<\sigma \leq \sigma_{0}$

$$
C(g, \sigma) \leq \tilde{C} g^{2}
$$

whith

$$
\tilde{C}=\frac{1}{m} \frac{1}{(2 \pi)^{2}}\left(\int \frac{|\rho|^{2}}{|k|} d^{3} k+1\right) .
$$

By (2.17) which also holds when $\rho$ is replaced by $\rho_{\sigma}$, we get that there exist two constants $b>0, a>0$ which do not depend on $\sigma \in\left(0, \sigma_{0}\right]$ and $g \in\left[-g_{1}, g_{1}\right]$ and satisfying $b g_{1}<1$ such that for $\Phi \in D\left(\tilde{H}_{0}\right)$ and for $\sigma \in\left(0, \sigma_{0}\right]$ with $\sigma_{0}$ sufficiently small

$$
\left\|\tilde{H}_{I, \sigma} \Phi\right\| \leq|g|\left(b\left\|\tilde{H}_{0} \Phi\right\|+a\|\Phi\|\right) .
$$

Therefore, since $\inf \sigma\left(\tilde{H}_{0}\right)=e(b, V)$, we obtain as a consequence of the KatoRellich theorem,

$$
\inf \sigma\left(\tilde{H}_{\sigma}\right) \geq e(b, V)-\max \left(\frac{a|g|}{1-b|g|}, a|g|+b|g||e(b, V)|\right) .
$$


Combining (4.2) and (4.3) we deduce the announced lower bound for $E_{\sigma}\left(P_{3}\right)$ with

$$
C=\max \left(\frac{a}{1-b g_{1}}, a+b|e(b, V)|\right)+\frac{g_{1}}{m} \frac{1}{(2 \pi)^{2}}\left(\int \frac{|\rho|^{2}}{|k|} d^{3} k+1\right) .
$$

Lemma 4.3. There exist $0<g_{2} \leq g_{1}$ and $\alpha>0$ such that

$$
E_{\sigma}\left(P_{3}-k_{3}\right)-E_{\sigma}\left(P_{3}\right) \geq-\frac{3}{4}|k|
$$

uniformly for $k \in \mathbb{R}^{3}, \sigma \in\left(0, \sigma_{0}\right],|g| \leq g_{2}$ and $\left|P_{3}\right| \leq \alpha$.

Remark that in this lemma we do not assume that $H_{\sigma}\left(P_{3}\right)$ has a ground state (i.e. we do not assume that $E_{\sigma}\left(P_{3}\right)$ is an eigenvalue of $\left.H_{\sigma}\left(P_{3}\right)\right)$ and actually we will use (4.4) in appendix A where we prove the existence of a ground state for the Hamiltonian with infrared cutoff.

Proof. First we remark that, if (4.4) is proved for $H_{\sigma}\left(P_{3}\right)+c$ for some constant $c$, it will hold also for $H_{\sigma}\left(P_{3}\right)$. Thus, in what follows, we suppose that $e(b, V)=0$.

The proof decomposes in two steps. In the first one, we consider the large values of $\left|k_{3}\right|$ (namely $\left|k_{3}\right| \geq m / 4$ ) while, in the second one, we consider the small values of $\left|k_{3}\right|$ (namely $\left|k_{3}\right| \leq m / 4$ ).

From (4.1), we deduce that, uniformly for $\sigma \in\left(0, \sigma_{0}\right]$ and $|g| \leq g_{1}$, we have for all $k$ and $P_{3}$

$$
E_{\sigma}\left(P_{3}-k_{3}\right)-E_{\sigma}\left(P_{3}\right) \geq-\frac{P_{3}^{2}}{2 m}-C|g|
$$

and thus assuming $\left|P_{3}\right| \leq \frac{\sqrt{3}}{4} m$ and $|g| \leq \frac{3 m}{32 C}$, (4.4) holds true for $\left|k_{3}\right| \geq m / 4$.

Now we suppose $\left|k_{3}\right| \leq m / 2$. As $E_{\sigma}\left(P_{3}-k_{3}\right)$ belongs to the spectrum of $H_{\sigma}\left(P_{3}-k_{3}\right)$ there exists a sequence $\left(\psi_{j}\right)_{j \geq 1}$ in $D\left(H_{\sigma}\left(P_{3}-k_{3}\right)\right)\left(=D\left(H_{0}\left(P_{3}=\right.\right.\right.$ $0))$ ) such that $\left\|\psi_{j}\right\|=1$ and

$$
\lim _{j \rightarrow \infty} H_{\sigma}\left(P_{3}-k_{3}\right) \psi_{j}-E_{\sigma}\left(P_{3}-k_{3}\right) \psi_{j}=0 .
$$

We then have for every $j$

$$
\begin{aligned}
\left\langle H_{\sigma}\left(P_{3}-k_{3}\right) \psi_{j}, \psi_{j}\right\rangle & =\left\langle H_{\sigma}\left(P_{3}\right) \psi_{j}, \psi_{j}\right\rangle+\frac{k_{3}^{2}}{2 m}-\frac{k_{3}}{m}\left\langle\left(P_{3}-d \Gamma\left(k_{3}\right)\right) \psi_{j}, \psi_{j}\right\rangle \\
& +\frac{2 g k_{3}}{m}\left\langle A_{3}\left(x^{\prime}, 0, \rho_{\sigma}\right) \psi_{j}, \psi_{j}\right\rangle \\
& \geq E_{\sigma}\left(P_{3}\right)+\frac{k_{3}^{2}}{2 m}-\frac{\left|k_{3}\right|}{m}\left|\left\langle\left(P_{3}-d \Gamma\left(k_{3}\right)\right) \psi_{j}, \psi_{j}\right\rangle\right| \\
& -\frac{2|g|\left|k_{3}\right|}{m}\left|\left\langle A_{3}\left(x^{\prime}, 0, \rho_{\sigma}\right) \psi_{j}, \psi_{j}\right\rangle\right| .
\end{aligned}
$$


In what follows $C$ will denote any positive constant which does not depend on $P_{3} \in \mathbb{R}, k_{3} \in \mathbb{R}, g \in\left[-g_{1}, g_{1}\right], \sigma \in\left(0, \sigma_{0}\right]$ and $j \geq 1$. We have

$$
\begin{aligned}
\left|\left\langle\left(P_{3}-d \Gamma\left(k_{3}\right)\right) \psi_{j}, \psi_{j}\right\rangle\right| & \leq\left|k_{3}\right|+\left|\left\langle\left(P_{3}-k_{3}-d \Gamma\left(k_{3}\right)\right) \psi_{j}, \psi_{j}\right\rangle\right| \\
& \leq\left|k_{3}\right|+\left\|\left(P_{3}-k_{3}-d \Gamma\left(k_{3}\right)\right) \psi_{j}\right\| \\
& \leq\left|k_{3}\right|+\left\|\left(P_{3}-k_{3}-d \Gamma\left(k_{3}\right)\right)^{2} \psi_{j}\right\|^{1 / 2} \\
& \leq\left|k_{3}\right|+\sqrt{2 m}\left\|H_{0}\left(P_{3}-k_{3}\right) \psi_{j}\right\|^{1 / 2} .
\end{aligned}
$$

On the other hand, we get from (2.10) and (2.11),

$$
\begin{aligned}
\mid\left\langle A_{3}\left(x^{\prime}, 0, \rho_{\sigma} \psi_{j}, \psi_{j}\right\rangle\right| & \leq C\left(\left\|H_{p h}^{1 / 2} \psi_{j}\right\|+1\right) \\
& \leq C\left(\left\|H_{0}\left(P_{3}-k_{3}\right) \psi_{j}\right\|^{1 / 2}+1\right) .
\end{aligned}
$$

Now, given $\epsilon>0$, let $J$ be such that

$$
\left\|H_{\sigma}\left(P_{3}-k_{3}\right) \psi_{j}-E_{\sigma}\left(P_{3}-k_{3}\right) \psi_{j}\right\| \leq \epsilon
$$

for every $j \geq J$ (notice that $J$ depends on $\epsilon$ but also on $\sigma$ and $P_{3}-k_{3}$ ).

Inserting (4.6) and (4.7) in (4.5) we obtain for $j \geq J$

$$
\begin{aligned}
E_{\sigma}\left(P_{3}-k_{3}\right)-E_{\sigma}\left(P_{3}\right) & \geq-\epsilon-\frac{k_{3}^{2}}{2 m}-\left|k_{3}\right| \sqrt{2 / m}\left\|H_{0}\left(P_{3}-k_{3}\right) \psi_{j}\right\|^{1 / 2} \\
& -\left|k_{3}\right| C|g|\left(\left\|H_{0}\left(P_{3}-k_{3}\right) \psi_{j}\right\|^{1 / 2}+1\right)
\end{aligned}
$$

and it remains to estimate $\left\|H_{0}\left(P_{3}-k_{3}\right) \psi_{j}\right\|$.

Writing

$H_{0}\left(P_{3}-k_{3}\right) \psi_{j}=\left(H_{\sigma}\left(P_{3}-k_{3}\right)-E_{\sigma}\left(P_{3}-k_{3}\right)\right) \psi_{j}+E_{\sigma}\left(P_{3}-k_{3}\right) \psi_{j}-H_{I, \sigma}\left(P_{3}-k_{3}\right) \psi_{j}$

we get for $j \geq J$

$$
\left\|H_{0}\left(P_{3}-k_{3}\right) \psi_{j}\right\| \leq \epsilon+\left|E_{\sigma}\left(P_{3}-k_{3}\right)\right|+\left\|H_{I, \sigma}\left(P_{3}-k_{3}\right) \psi_{j}\right\| .
$$

Using (2.17) there exists $C>0$ such that

$$
\left\|H_{I, \sigma}\left(P_{3}\right) \phi\right\| \leq|g| C\left(\left\|H_{0}\left(P_{3}\right) \phi\right\|+1\right)
$$

for every $P_{3} \in \mathbb{R}$ and $\phi \in D\left(H_{0}\left(P_{3}\right)\right)$, $\|\phi\| \leq 1$. Thus, choosing $g_{1}^{\prime} \leq g_{1}$ such that $g_{1}^{\prime} C \leq 1 / 2$, we get

$$
\left\|H_{0}\left(P_{3}-k_{3}\right) \psi_{j}\right\| \leq 2 \epsilon+2\left|E_{\sigma}\left(P_{3}-k_{3}\right)\right|+2|g| C
$$

for $j \geq J$ and $|g| \leq g_{1}^{\prime}$. Inserting this last inequality in (4.9) we obtain

$$
\begin{aligned}
E_{\sigma}\left(P_{3}-k_{3}\right)-E_{\sigma}\left(P_{3}\right) & \geq-\epsilon-\frac{k_{3}^{2}}{2 m}-2\left|k_{3}\right| \sqrt{1 / m}\left(\epsilon+\left|E_{\sigma}\left(P_{3}-k_{3}\right)\right|+|g| C\right)^{1 / 2} \\
& -\left|k_{3}\right| C|g|\left(\left(2 \epsilon+2\left|E_{\sigma}\left(P_{3}-k_{3}\right)\right|+2|g| C\right)^{1 / 2}+1\right)
\end{aligned}
$$


for every $\epsilon>0$. Hence

$$
\begin{gathered}
E_{\sigma}\left(P_{3}-k_{3}\right)-E_{\sigma}\left(P_{3}\right) \geq-\left|k_{3}\right|\left\{\frac{\left|k_{3}\right|}{2 m}+2 \sqrt{1 / m}\left(\left|E_{\sigma}\left(P_{3}-k_{3}\right)\right|+|g| C\right)^{1 / 2}\right. \\
\left.+C|g|\left(\left(2\left|E_{\sigma}\left(P_{3}-k_{3}\right)\right|+2|g| C\right)^{1 / 2}+1\right)\right\}
\end{gathered}
$$

for every $k_{3}$ and $P_{3}$ in $\mathbb{R}$. Finally we use (4.1) to get for $\left|k_{3}\right| \leq m / 4$,

$$
\left|E_{\sigma}\left(P_{3}-k_{3}\right)\right| \leq C|g|+\frac{P_{3}^{2}}{2 m}+\frac{\left|P_{3}\right|}{4}+\frac{m}{32}
$$

and therefore there exit $\alpha>0$ and $g_{2} \leq g_{1}^{\prime}$ such that for $\left|P_{3}\right| \leq \alpha,\left|k_{3}\right| \leq m / 4$ and $|g| \leq g_{2}$,

$$
E_{\sigma}\left(P_{3}-k_{3}\right)-E_{\sigma}\left(P_{3}\right) \geq-\frac{3}{4}\left|k_{3}\right|
$$

\subsection{Proof of (iii) of theorem 4.1}

In this section we assume that assertion (ii) of theorem 4.1 is already proved (see appendix A). Thus let $\Phi_{\sigma}\left(P_{3}\right)$ denote a normalized ground state of $H_{\sigma}\left(P_{3}\right)$, i.e.

$$
H_{\sigma}\left(P_{3}\right) \Phi_{\sigma}\left(P_{3}\right)=E_{\sigma}\left(P_{3}\right) \Phi_{\sigma}\left(P_{3}\right) .
$$

The main problem in proving (iii) of theorem 4.1 is to control the number of photons in the ground state $\Phi_{\sigma}\left(P_{3}\right)$ uniformly with respect to $\sigma$. The operator number of photons $N_{p h}$ is given by

$$
N_{p h}:=\sum_{\mu=1,2} \int_{\mathbb{R}^{3}} d^{3} k a_{\mu}^{*}(k) a_{\mu}(k)
$$

and we set

$$
G(k):=|k|^{1 / 2}|\rho(k)|+\frac{|\rho(k)|}{|k|^{1 / 2}} .
$$

Lemma 4.4. There exists a constant $C$ independent of $g$ and $\sigma$ such that

$$
\left\|\left(I \otimes N_{p h}^{1 / 2}\right) \Phi_{\sigma}\left(P_{3}\right)\right\| \leq C|g|\left(\int \frac{|G(k)|^{2}}{|k|^{2}} d^{3} k\right)^{1 / 2}
$$

for every $\sigma \in\left(0, \sigma_{0}\right],|g| \leq g_{2}$ and $\left|P_{3}\right| \leq \alpha$ ( $g_{2}$ and $\alpha$ are introduced in lemma 4.3).

Proof. One easily verifies that one has the following "pull through" formula

$$
a_{\mu}(k) H_{\sigma}\left(P_{3}\right)=H_{\sigma}\left(P_{3}-k_{3}\right) a_{\mu}(k)+\omega(k) a_{\mu}(k)+v_{\mu}(k)
$$


with

$$
\begin{aligned}
v_{\mu}(k) & =\frac{i g}{2 \pi m}|k|^{1 / 2} \rho_{\sigma}(k) e^{-i k^{\prime} \cdot x^{\prime}}\left(\frac{k}{|k|} \wedge \epsilon_{\mu}(k)\right) \\
& -\sum_{j=1,2} \frac{g}{2 \pi m} \frac{\rho_{\sigma}(k)}{|k|^{1 / 2}} e^{-i k^{\prime} \cdot x^{\prime}} \epsilon_{\mu}(k)_{j}\left(p_{j}-e a_{j}\left(x^{\prime}\right)\right) \\
& -\frac{g}{2 \pi m} \frac{\rho_{\sigma}(k)}{|k|^{1 / 2}} e^{-i k^{\prime} \cdot x^{\prime}} \epsilon_{\mu}(k)_{3}\left(P_{3}-d \Gamma\left(k_{3}\right)\right) \\
& +\frac{g^{2}}{2 \pi m} \frac{\rho_{\sigma}(k)}{|k|^{1 / 2}} e^{-i k^{\prime} \cdot x^{\prime}} \epsilon_{\mu}(k) \cdot A\left(x^{\prime}, 0, \rho_{\sigma}\right) .
\end{aligned}
$$

Applying (4.13) to $\Phi_{\sigma}\left(P_{3}\right)$, we obtain

$$
\begin{aligned}
& 0=\left(H_{\sigma}\left(P_{3}-k_{3}\right)-E_{\sigma}\left(P_{3}\right)+\omega(k)\right) a_{\mu}(k) \Phi_{\sigma}\left(P_{3}\right)+v_{\mu}(k) \Phi_{\sigma}\left(P_{3}\right) \\
& =\left(H_{\sigma}\left(P_{3}-k_{3}\right)-E_{\sigma}\left(P_{3}-k_{3}\right)+E_{\sigma}\left(P_{3}-k_{3}\right)-E_{\sigma}\left(P_{3}\right)+\omega(k)\right) a_{\mu}(k) \Phi_{\sigma}\left(P_{3}\right) \\
& \quad+v_{\mu}(k) \Phi_{\sigma}\left(P_{3}\right) \\
& \quad \text { and thus, as } H_{\sigma}\left(P_{3}-k_{3}\right)-E_{\sigma}\left(P_{3}-k_{3}\right) \geq 0, \text { we get using }(4.4), \\
& \quad\left\|a_{\mu}(k) \Phi_{\sigma}\left(P_{3}\right)\right\| \leq \frac{1}{\mid E_{\sigma}\left(P_{3}-k_{3}\right)-E_{\sigma}\left(P_{3}\right)+\omega\left(P_{3}\right) \|} \\
& \quad \leq \frac{4}{|k|}\left\|v_{\mu}(k) \Phi_{\sigma}\left(P_{3}\right)\right\|
\end{aligned}
$$

for $\left|P_{3}\right| \leq \alpha$ and $|g| \leq g_{2}$. Using estimates from section 2.4 (and similarly as $(2.17))$ we show that there exists a constant $C>0$ such that

$$
\left\|v_{\mu}(k) \Phi_{\sigma}\left(P_{3}\right)\right\| \leq C|g| G(k)\left(\left\|\left(H_{0}\left(P_{3}\right)-e(b, V)\right) \Phi_{\sigma}\left(P_{3}\right)\right\|+1\right) .
$$

Now, similarly as (4.10), we have for $|g| \leq g_{2}$

$$
\left\|H_{0}\left(P_{3}\right) \Phi_{\sigma}\left(P_{3}\right)\right\| \leq 2\left|E_{\sigma}\left(P_{3}\right)\right|+2 C g .
$$

By lemma $4.2\left|E_{\sigma}\left(P_{3}\right)\right| \leq C|g|+\frac{P_{3}^{2}}{2 m}$ and therefore we deduce from (4.14) that

$$
\left\|a_{\mu}(k) \Phi_{\sigma}\left(P_{3}\right)\right\| \leq C|g| \frac{G(k)}{|k|}
$$

where the constant $C$ is uniform with respect to $\left|P_{3}\right| \leq \alpha, \sigma \in\left(0, \sigma_{0}\right]$ and $|g| \leq g_{2}$.

Thus lemma 4.4 follows from this last inequality and from

$$
\left\|\left(I \otimes N_{p h}^{1 / 2}\right) \Phi_{\sigma}\left(P_{3}\right)\right\|^{2}=\sum_{\mu=1,2} \int d^{3} k\left\|\left(I \otimes a_{\mu}(k)\right) \Phi_{\sigma}\left(P_{3}\right)\right\|^{2} .
$$

Let us remark that the above proof is a little bit formal since we do not check that $\Phi_{\sigma}\left(P_{3}\right)$ belongs to the domain of the different operators involved in the 
pull through formula (4.13). But by mimicking [29] one easily gets a rigourous proof. We omit the details.

Recall that we denote by $P_{(.]}$the spectral measure of $h(b, V)$ and by $P_{\Omega_{p h}}$ the orthogonal projection on $\Omega_{p h}$. We have the following

Lemma 4.5. Fix $\lambda \in(e(b, V), 0)$. There exists $\delta_{g}(\lambda)>0$ such that $\delta_{g}(\lambda) \rightarrow 0$ when $g \rightarrow 0$ and

$$
\left\langle P_{[\lambda, \infty)} \otimes P_{\Omega_{p h}} \Phi_{\sigma}\left(P_{3}\right), \Phi_{\sigma}\left(P_{3}\right)\right\rangle \leq \delta_{g}(\lambda)
$$

for every $\sigma \in\left(0, \sigma_{0}\right],\left|P_{3}\right| \leq \alpha$ and $|g| \leq g_{2}$.

Proof. Since $P_{\Omega_{p h}} H_{p h}=0$ and $P_{\Omega_{p h}}\left(P_{3}-d \Gamma\left(k_{3}\right)\right)^{2}=P_{3}^{2} P_{\Omega_{p h}}$ we get

$$
\begin{aligned}
& \left(P_{[\lambda, \infty)} \otimes P_{\Omega_{p h}}\right)\left(H_{\sigma}\left(P_{3}\right)-E_{\sigma}\left(P_{3}\right)\right)=P_{[\lambda, \infty)}(h(b, V) \otimes I) \otimes P_{\Omega_{p h}} \\
& +\left(\frac{P_{3}^{2}}{2 m}-E_{\sigma}\left(P_{3}\right)\right) P_{[\lambda, \infty)} \otimes P_{\Omega_{p h}}+P_{[\lambda, \infty)} \otimes P_{\Omega_{p h}} H_{I, \sigma}\left(P_{3}\right) .
\end{aligned}
$$

Applying this last equality to $\Phi_{\sigma}\left(P_{3}\right)$ we obtain

$$
\begin{aligned}
0 & =P_{[\lambda, \infty)}(h(b, V) \otimes I) \otimes P_{\Omega_{p h}} \Phi_{\sigma}\left(P_{3}\right) \\
& +\left(\frac{P_{3}^{2}}{2 m}-E_{\sigma}\left(P_{3}\right)\right) P_{[\lambda, \infty)} \otimes P_{\Omega_{p h}} \Phi_{\sigma}\left(P_{3}\right) \\
& +P_{[\lambda, \infty)} \otimes P_{\Omega_{p h}} H_{I, \sigma}\left(P_{3}\right) \Phi_{\sigma}\left(P_{3}\right) .
\end{aligned}
$$

Since $h(b, V) P_{[\lambda, \infty)} \geq \lambda P_{[\lambda, \infty)}$ we obtain from (4.17) and lemma 4.2

$$
\begin{aligned}
& \left\langle P_{[\lambda, \infty)} \otimes P_{\Omega_{p h}} \Phi_{\sigma}\left(P_{3}\right), \Phi_{\sigma}\left(P_{3}\right)\right\rangle \leq \\
& \frac{-1}{\lambda-e(b, V)}\left\langle\left(P_{[\lambda, \infty)} \otimes P_{\Omega_{p h}}\right) H_{I, \sigma}\left(P_{3}\right) \Phi_{\sigma}\left(P_{3}\right), \Phi_{\sigma}\left(P_{3}\right)\right\rangle
\end{aligned}
$$

for every $\sigma \in\left(0, \sigma_{0}\right]$. The lemma then follows from (2.17) (which is also valid for $\left.H_{I, \sigma}\left(P_{3}\right)\right)$.

We are now able to conclude the proof of (iii) of theorem 4.1. We have

$$
\begin{gathered}
\left\langle P_{(-\infty, \lambda]} \otimes P_{\Omega_{p h}} \Phi_{\sigma}\left(P_{3}\right), \Phi_{\sigma}\left(P_{3}\right)\right\rangle=1 \\
-\left\langle P_{[\lambda, \infty)} \otimes P_{\Omega_{p h}} \Phi_{\sigma}\left(P_{3}\right), \Phi_{\sigma}\left(P_{3}\right)\right\rangle \\
-\left\langle 1 \otimes P_{\Omega_{p h}}^{\perp} \Phi_{\sigma}\left(P_{3}\right), \Phi_{\sigma}\left(P_{3}\right)\right\rangle .
\end{gathered}
$$

Now it suffices to remark that the second term in the right hand side of this equality is estimated by lemma 4.5 and, noticing that $\left\|P_{\Omega_{p h}}^{\perp} \phi\right\| \leq\left\|N_{p h}^{1 / 2} \phi\right\|$, the third term is estimated by lemma 4.4 . 


\section{A Exitences of a ground state for the Hamilto- nian with infrared cutoff}

In this appendix we prove the assertion (ii) of theorem 4.1 : for $\sigma$ and $P_{3}$ small enough, the Hamiltonian with infrared cutoff has a ground state. This result is not surprising but the complete proof is long. Actually it follows by mimicking $[20,21,16]$ (see also [34]) and, here, we only give a sketch of the proof.

In this appendix we are faced with the lack of smoothness of the $\epsilon_{\mu}(k)$ 's which define vector fields on spheres $|k|=$ cst (see [33, 23]). It suffices to consider one example. From now on suppose that

$$
\epsilon_{1}(k)=\frac{1}{\sqrt{k_{1}^{2}+k_{2}^{2}}}\left(k_{2},-k_{1}, 0\right) \quad \text { and } \quad \epsilon_{2}(k)=\frac{k}{|k|} \wedge \epsilon_{1}(k) .
$$

The functions $\epsilon_{\mu}(k), \mu=1,2$, are smooth only on $\mathbb{R}^{3} \backslash\left\{\left(0,0, k_{3}\right) \mid k_{3} \in \mathbb{R}\right\}$. Nevertheless, in our case, we can overcome this problem easily by choosing the regularization $\rho_{\sigma}$ of $\rho$ as a $C^{\infty}$ function whose support does not intersect the line $\left\{\left(0,0, k_{3}\right) \mid k_{3} \in \mathbb{R}\right\}$. From now on we suppose that it is the case.

Let $\omega_{\bmod }(k)$ be the modified dispersion relation as defined in ([21], section 5 , hypothesis 3$)$, i.e. : $\omega_{\bmod }(k)$ is a smooth function satisfying

(i) $\omega_{\bmod }(k) \geq \max \left(|k|, \frac{\sigma}{2}\right)$ for all $k \in \mathbb{R}^{3}, \omega_{\bmod }(k)=|k|$ for $|k| \geq \sigma$.

(ii) $\left|\nabla \omega_{\bmod }(k)\right| \leq 1$ for all $k \in \mathbb{R}^{3}$, and $\nabla \omega_{\bmod }(k) \neq 0$ unless $k=0$.

(iii) $\omega_{\bmod }\left(k_{1}+k_{2}\right) \leq \omega_{\bmod }\left(k_{1}\right)+\omega_{\bmod }\left(k_{2}\right)$ for all $k_{1}, k_{2} \in \mathbb{R}^{3}$.

We set

$$
H_{p h, \bmod }=\sum_{\mu=1,2} \int \omega_{\bmod }(k) a_{\mu}^{\star}(k) a_{\mu}(k) d^{3} k
$$

and

$$
H_{\text {mod }, \sigma}\left(P_{3}\right)=h(b, V) \otimes I+I \otimes\left\{\frac{1}{2 m}\left(P_{3}-d \Gamma\left(k_{3}\right)\right)^{2}+H_{p h, \bmod }\right\}+H_{I, \sigma}\left(P_{3}\right) .
$$

Theorem 2.4, with the same assumption (2.18), is still valid for $H_{\bmod , \sigma}\left(P_{3}\right)$. Set $E_{\text {mod }, \sigma}\left(P_{3}\right):=\inf \sigma\left(H_{\text {mod }, \sigma}\left(P_{3}\right)\right)$. Then $E_{\text {mod, } \sigma}\left(P_{3}\right)$ still satisfies lemma 4.3 and (4.4) for the same constants $g_{2}$ and $\alpha$. Moreover, according to ([21]; thm 3), $E_{\sigma}\left(P_{3}\right)=E_{\bmod , \sigma}\left(P_{3}\right)$ for $\left|P_{3}\right| \leq \alpha$ and $|g| \leq g_{2}$ and $E_{\sigma}\left(P_{3}\right)$ is an eigenvalue of $H_{\sigma}\left(P_{3}\right)$ if and only if $E_{\text {mod, } \sigma}\left(P_{3}\right)$ is an eigenvalue of $H_{\text {mod, } \sigma}\left(P_{3}\right)$. Thus in order to prove that $H_{\sigma}\left(P_{3}\right)$ has a ground state it suffices to prove that $E_{\text {mod }, \sigma}\left(P_{3}\right)<\inf \sigma_{\text {ess }}\left(H_{\text {mod }, \sigma}\left(P_{3}\right)\right)$. The proof is by contradiction, so we suppose that $E_{\bmod , \sigma}\left(P_{3}\right)=\inf \sigma_{\mathrm{ess}}\left(H_{\bmod , \sigma}\left(P_{3}\right)\right)$ and we set

$$
\lambda=E_{\bmod , \sigma}\left(P_{3}\right)=\inf \sigma_{\mathrm{ess}}\left(H_{\mathrm{mod}, \sigma}\left(P_{3}\right)\right) .
$$

We now observe that $E_{\text {mod, } \sigma}\left(P_{3}\right)$ satisfies (4.1) for $|g| \leq g_{2}$.

Let $\delta:=\operatorname{dist}(e(v, V), \sigma(h(b, V)) \backslash\{e(b, V)\})>0$. According to (4.1) there 
exist $0<\beta \leq \alpha$ and $0<g_{3} \leq g_{2}$ such that

$$
\begin{aligned}
& \lambda \leq e(b, V)+\frac{\delta}{3} \quad \text { for }\left|P_{3}\right| \leq \beta \\
& C|g| \leq \frac{\delta}{12} \quad \text { for }|g| \leq g_{3}
\end{aligned}
$$

where $C$ is the constant in (4.1).

Let $\Delta$ be an interval such that $\lambda \in \Delta$ and $\sup \Delta<e(b, V)+\frac{\delta}{2}$. Thus

$$
e(b, V)+\frac{2 \delta}{3}-\sup \Delta-C|g| \geq \frac{\delta}{12}
$$

for $\left|P_{3}\right| \leq \beta$ and $|g| \leq g_{3}$ and we introduce $\eta>0$ such that

$$
\eta^{2}<e(b, V)+\frac{2 \delta}{3}-\sup \Delta-C|g| .
$$

Then, along the same lines as in the proof of theorem II.1 in [8], one easily shows that it exists $M_{\Delta}$ such that for any $\left|P_{3}\right| \leq \beta$ and any $|g| \leq g_{3}$

$$
\left\|\left(e^{\eta\left|x^{\prime}\right|} \otimes I\right) \chi_{\Delta}\left(H_{\bmod , \sigma}\left(P_{3}\right)\right)\right\| \leq M_{\Delta} .
$$

Since we assume $\lambda \in \sigma_{\text {ess }}\left(H_{\text {mod }, \sigma}\left(P_{3}\right)\right)$ there exits a sequence $\left(\phi_{n}\right)_{n \geq 1}$, with $\left\|\phi_{n}\right\|=1$, such that

$$
\begin{aligned}
& \phi_{n} \in \operatorname{Ran} \chi_{\Delta}\left(H_{\bmod , \sigma}\left(P_{3}\right)\right), \\
& \left(H_{\bmod , \sigma}\left(P_{3}\right)-\lambda\right) \phi_{n} \rightarrow_{n \rightarrow 0} 0, \\
& w-\lim _{n \rightarrow 0} \phi_{n}=0
\end{aligned}
$$

and therefore

$$
\lambda=\lim _{n \rightarrow \infty}\left\langle\phi_{n}, H_{\bmod , \sigma}\left(P_{3}\right) \phi_{n}\right\rangle .
$$

Notice that, as for (A.3), one easily shows that for any $\left|P_{3}\right| \leq \beta$, any $|g| \leq g_{3}$ and any $n \geq 1$

$$
\begin{aligned}
& \left\|\left(e^{\frac{\eta}{2}\left|x^{\prime}\right|} \otimes I\right) \chi_{\Delta}\left(H_{\bmod , \sigma}\left(P_{3}\right)\right) \nabla_{x^{\prime}} \phi_{n}\right\| \leq M_{\Delta}^{\prime} \\
& \left\|\left(e^{\frac{\eta}{2}\left|x^{\prime}\right|} \otimes I\right) \chi_{\Delta}\left(H_{\bmod , \sigma}\left(P_{3}\right)\right) d \Gamma\left(k_{3}\right) \phi_{n}\right\| \leq M_{\Delta}^{\prime}
\end{aligned}
$$

where $M_{\Delta}^{\prime}$ is a finite constant.

Now, in order to estimate $\left\langle\phi_{n}, H_{\bmod , \sigma}\left(P_{3}\right) \phi_{n}\right\rangle$ from below, we need to localize the photons. Let $j_{0}, j_{\infty} \in C^{\infty}\left(\mathbb{R}^{3}\right)$ be real valued functions with $j_{0}^{2}+j_{\infty}^{2}=1$, $j_{0}(y)=1$ for $|y| \leq 1$ and $j_{0}(y)=0$ for $|y| \geq 2$. Given $R>0$, we set $j_{\cdot, R}(y)=$ $j$. $\left(\frac{y}{R}\right)$ and let $j_{R}=\left(j_{0, R}, j_{\infty, R}\right)$. Here $y=\frac{1}{i} \nabla_{k}$ and $j_{R}$ is an operator from $\mathcal{F}$ to $\mathcal{F} \otimes \mathcal{F}$.

Then let $\check{\Gamma}\left(j_{R}\right), d \check{\Gamma}\left(j_{R}, \omega_{\bmod } j_{R}-j_{R} \omega_{\bmod }\right)$ be the operators from $L^{2}\left(\mathbb{R}^{3}, \mathbb{C}^{2}\right) \otimes$ $\mathcal{F}$ to $L^{2}\left(\mathbb{R}^{3}, \mathbb{C}^{2}\right) \otimes \mathcal{F} \otimes \mathcal{F}$ as defined in sections 2.13 and 2.14 of [16] (see also section 2.6 of $[21])$. Here $\underline{\omega_{\bmod }}:=\left(\omega_{\bmod }, \omega_{\text {mod }}\right)$. Roughly speaking, $\check{\Gamma}\left(j_{R}\right)$ 
separates the set of photons between photons localized around the electron and photons that escape to infinity (when $R \rightarrow \infty$ ).

Set

$$
\begin{aligned}
& G_{l, \mu}\left(x^{\prime}, \rho_{\sigma}\right)=\frac{1}{2 \pi} \frac{\rho_{\sigma}}{|k|^{1 / 2}} e^{-i k x^{\prime}} \epsilon_{\mu}(k)_{l}, \quad l=1,2,3, \\
& H_{\mu}\left(x^{\prime}, \rho_{\sigma}\right)=-\frac{i}{2 \pi}|k|^{1 / 2} \rho_{\sigma}(k) \sigma \cdot\left(\frac{k}{|k|} \wedge \epsilon_{\mu}(k)\right) e^{-i k x^{\prime}}, \\
& \Phi_{\mu}(h)=\frac{1}{\sqrt{2}}\left(a_{\mu}(h)+a_{\mu}^{\star}(h)\right) .
\end{aligned}
$$

Let $\check{H}_{\text {mod }, \sigma}\left(P_{3}\right)$ be the following operator in $L^{2}\left(\mathbb{R}^{3}, \mathbb{C}^{2}\right) \otimes \mathcal{F} \otimes \mathcal{F}$ :

$$
\begin{aligned}
& \check{H}_{\text {mod, }, \sigma}\left(P_{3}\right)=h(b, V) \otimes I \otimes I+I \otimes H_{\text {mod }, p h} \otimes I+I \otimes I \otimes H_{\text {mod }, p h} \\
& +\frac{1}{2 m} I \otimes\left(P_{3}-I \otimes d \Gamma\left(k_{3}\right) \otimes I-I \otimes I \otimes d \Gamma\left(k_{3}\right)\right)^{2} \\
& -\frac{g}{2 m} \sqrt{2} \sum_{\mu=1,2} \Phi_{\mu}\left(H_{\mu}\left(x^{\prime}, \rho_{\sigma}\right)\right) \otimes I \\
& -\frac{g}{m} \sqrt{2} \sum_{\mu=1,2} \sum_{j=1,2}\left(p_{j}-e a_{j}\left(x^{\prime}\right)\right) \Phi_{\mu}\left(G_{j, \mu}\left(x^{\prime}, \rho_{\sigma}\right)\right) \otimes I \\
& -\frac{g}{m} \sqrt{2}\left(P_{3}-I \otimes d \Gamma\left(k_{3}\right) \otimes I-I \otimes I \otimes d \Gamma\left(k_{3}\right)\right)\left(\sum_{\mu=1,2} \Phi_{\mu}\left(G_{3, \mu}\left(x^{\prime}, \rho_{\sigma}\right)\right) \otimes I\right) \\
& +\frac{g^{2}}{2 m} \sum_{l=1,2,3}\left(\sum_{\mu=1,2} \Phi_{\mu}\left(G_{l, \mu}\left(x^{\prime}, \rho_{\sigma}\right)\right)\right)^{2} \otimes I .
\end{aligned}
$$

Again, assuming (2.18), $\check{H}_{\text {mod, } \sigma}\left(P_{3}\right)$ is a self-adjoint operator in $L^{2}\left(\mathbb{R}^{3}, \mathbb{C}^{2}\right) \otimes$ $\mathcal{F} \otimes \mathcal{F}$ for $|g| \leq g_{1}$. We remark that

$$
\check{H}_{\bmod , \sigma}\left(P_{3}\right) \geq E_{\bmod , \sigma}\left(P_{3}\right)+I \otimes I \otimes H_{\bmod , p h}
$$

and thus $\left\langle\phi, \check{H}_{\bmod , \sigma}\left(P_{3}\right) \phi\right\rangle \geq E_{\bmod , \sigma}\left(P_{3}\right)+\frac{\sigma}{2}$ if the state $\phi$ has a component along the delocalized photons. Actually we are going to prove that, since $\lambda$ is in the essential spectrum, $\check{\Gamma}\left(j_{R}\right) \phi_{n}$ has a non vanishing component along the delocalized photons and thus, in view of (A.4), we will obtain a contradiction with (A.1).

Finally set for $l=1,2,3$

$T_{l, R}(G)\left(x^{\prime}\right)=\sum_{\mu=1,2}\left(\Phi_{\mu}\left(\left(j_{0, R}-1\right) G_{l, \mu}\left(x^{\prime}, \rho_{\sigma}\right)\right) \otimes 1+1 \otimes \Phi_{\mu}\left(j_{\infty, R} G_{l, \mu}\left(x^{\prime}, \rho_{\sigma}\right)\right)\right)$

and

$$
T_{R}(H)\left(x^{\prime}\right)=\sum_{\mu=1,2}\left(\Phi_{\mu}\left(\left(j_{0, R}-1\right) H_{\mu}\left(x^{\prime}, \rho_{\sigma}\right)\right) \otimes 1+1 \otimes \Phi_{\mu}\left(j_{\infty, R} H_{\mu}\left(x^{\prime}, \rho_{\sigma}\right)\right)\right) .
$$


By using [16] (sections 2.13 and 2.14) and [21] (sections 2.5 and 2.6) we obtain that $\check{H}_{\text {mod, } \sigma}\left(P_{3}\right)$ and $H_{\text {mod, } \sigma}\left(P_{3}\right)$ are almost conjugated by $\check{\Gamma}\left(j_{R}\right)$, namely

$$
\begin{aligned}
\check{\Gamma}\left(j_{R}\right) & H_{\text {mod, },}\left(P_{3}\right)-\check{H}_{\text {mod, },}\left(P_{3}\right) \check{\Gamma}\left(j_{R}\right)=-\frac{g}{2 m} \sqrt{2} T_{R}(H)\left(x^{\prime}\right) \check{\Gamma}\left(j_{R}\right) \\
- & \frac{g}{2 m} \sqrt{2}\left(P_{3}-I \otimes d \Gamma\left(k_{3}\right) \otimes I-I \otimes I \otimes d \Gamma\left(k_{3}\right)\right) T_{3 R}(G)\left(x^{\prime}\right) \check{\Gamma}\left(j_{R}\right) \\
- & \frac{g}{m} \sqrt{2} \sum_{j=1,2}\left(p_{j}-e a_{j}\left(x^{\prime}\right)\right) T_{j R}(G)\left(x^{\prime}\right) \check{\Gamma}\left(j_{R}\right)-d \check{\Gamma}\left(j_{R}, \underline{\omega_{\text {mod }}} j_{R}-j_{R} \omega_{\text {mod }}\right) \\
- & d \check{\Gamma}\left(j_{R}, \underline{k}_{3} j_{R}-j_{R} k_{3}\right)\left(\frac{1}{2 m}\left(P_{3}-d \Gamma\left(k_{3}\right)\right)+\sqrt{2} \sum_{\mu=1,2} \Phi_{\mu}\left(G_{3, \mu}\left(x^{\prime}, \rho_{\sigma}\right)\right)\right) \\
& -\frac{1}{2 m}\left(P_{3}-I \otimes d \Gamma\left(k_{3}\right) \otimes I-I \otimes I \otimes d \Gamma\left(k_{3}\right)\right) d \check{\Gamma}\left(j_{R}, \underline{k_{3}} j_{R}-j_{R} k_{3}\right) \\
+ & \frac{g^{2}}{2 m}\left[\sum_{l=1,2,3} \sum_{\mu=1,2} \sum_{\mu^{\prime}=1,2}\right. \\
& \left\{a_{\mu}\left(j_{0, R} G_{l, \mu}\left(x^{\prime}, \rho_{\sigma}\right)\right) \otimes 1+1 \otimes a_{\mu}\left(j_{\infty, R} G_{l, \mu}\left(x^{\prime}, \rho_{\sigma}\right)\right)\right. \\
+ & \left.a_{\mu}^{\star}\left(j_{0, R} G_{l, \mu}\left(x^{\prime}, \rho_{\sigma}\right)\right) \otimes 1+1 \otimes a_{\mu}^{\star}\left(j_{\infty, R} G_{l, \mu}\left(x^{\prime}, \rho_{\sigma}\right)\right)\right\} \\
& \left\{a_{\mu^{\prime}}\left(j_{0, R} G_{l, \mu^{\prime}}\left(x^{\prime}, \rho_{\sigma}\right)\right) \otimes 1+1 \otimes a_{\mu^{\prime}}\left(j_{\infty, R} G_{l, \mu^{\prime}}\left(x^{\prime}, \rho_{\sigma}\right)\right)\right. \\
+ & \left.a_{\mu^{\prime}}^{\star}\left(j_{0, R} G_{l, \mu^{\prime}}\left(x^{\prime}, \rho_{\sigma}\right)\right) \otimes 1+1 \otimes a_{\mu^{\prime}}^{\star}\left(j_{\infty, R} G_{l, \mu^{\prime}}\left(x^{\prime}, \rho_{\sigma}\right)\right)\right\} \\
& \left.-\sum_{l=1,2,3}\left(\sum_{\mu=1,2} \Phi_{\mu}\left(G_{l, \mu}\left(x^{\prime}, \rho_{\sigma}\right)\right)\right)^{2} \otimes I\right] \check{\Gamma}\left(j_{R}\right) .
\end{aligned}
$$

Since $\rho_{\sigma}$ is a $C_{0}^{\infty}$ function, one has for $\gamma>0$

$$
\begin{aligned}
& \left(1-\Delta_{k^{\prime}}\right)^{\gamma} \frac{\rho_{\sigma}(k)}{|k|^{1 / 2}} \epsilon_{\mu}(k)_{l} \in L^{2}\left(\mathbb{R}^{3}\right) \quad l=1,2,3, \mu=1,2, \sigma>0 \\
& \left(1-\Delta_{k^{\prime}}\right)^{\gamma} \rho_{\sigma}(k)|k|^{1 / 2} \sigma \cdot\left(\frac{k}{|k|} \wedge \epsilon_{\mu}(k)\right) \in L^{2}\left(\mathbb{R}^{3}\right) \quad \mu=1,2, \sigma>0 .
\end{aligned}
$$

Here $k^{\prime}=\left(k_{1}, k_{2}\right)$.

We then prove, as in ([21] lemma 9), that both

$$
e^{-\frac{\eta}{2}\left|x^{\prime}\right|} T_{l, R}(G)\left(x^{\prime}\right)\left(I+I \otimes N_{p h} \otimes I+I \otimes I \otimes N_{p h}\right)^{-1 / 2}
$$

and

$$
e^{-\frac{\eta}{2}\left|x^{\prime}\right|} T_{R}(H)\left(x^{\prime}\right)\left(I+I \otimes N_{p h} \otimes I+I \otimes I \otimes N_{p h}\right)^{-1 / 2}
$$

tend to zero in $L^{2}\left(\mathbb{R}^{3}, \mathbb{C}^{2}\right) \otimes \mathcal{F} \otimes \mathcal{F}$ when $R \rightarrow \infty$. Therefore it follows from (A.3) and (A.7) that

$$
\left\langle\phi_{n}, H_{\mathrm{mod}, \sigma}\left(P_{3}\right) \phi_{n}\right\rangle=\left\langle\phi_{n}, \check{\Gamma}\left(j_{R}\right)^{\star} \check{H}_{\mathrm{mod}, \sigma}\left(P_{3}\right) \check{\Gamma}\left(j_{R}\right) \phi_{n}\right\rangle+o\left(R^{0}\right)
$$


uniformly in $n$ (cf. [21] and [16]).

Denoting by $P_{\Omega_{\infty}}$ the orthogonal projection on the vacuum of delocalized photons, we have using (A.6)

$$
\begin{aligned}
\left\langle\phi_{n}, \check{\Gamma}\left(j_{R}\right)^{\star} \check{H}_{\bmod , \sigma}\left(P_{3}\right) \check{\Gamma}\left(j_{R}\right) \phi_{n}\right\rangle & \geq E_{\bmod , \sigma}\left(P_{3}\right)+\frac{\sigma}{2} \\
& -\frac{\sigma}{2}\left\langle\phi_{n}, \check{\Gamma}\left(j_{R}\right)^{\star}\left(I \otimes I \otimes P_{\Omega_{\infty}}\right) \check{\Gamma}\left(j_{R}\right) \phi_{n}\right\rangle .
\end{aligned}
$$

On the other hand we verify

$$
\check{\Gamma}\left(j_{R}\right)^{\star}\left(I \otimes I \otimes P_{\Omega_{\infty}}\right) \check{\Gamma}\left(j_{R}\right)=\Gamma\left(j_{0, R}^{2}\right) .
$$

Then, by using lemma 4.2 for $E_{\bmod , \sigma}\left(P_{3}\right)$ and the compactness of $\chi_{\Delta}\left(H_{\bmod , \sigma}\left(P_{3}\right)\right) e^{-\eta\left|x^{\prime}\right|} \Gamma\left(j_{0, R}^{2}\right)\left(H_{\bmod , \sigma}\left(P_{3}\right)+i\right)^{-1}$ (see [16] lemma 34 or [21] lemma 36 and [7] theorem 2.6), we deduce from (A.8), letting $n \rightarrow \infty$,

$$
\lambda \geq E_{\bmod , \sigma}\left(P_{3}\right)+\frac{\sigma}{2}+o\left(R^{0}\right) .
$$

Letting $R \rightarrow \infty$ we get a contradiction with (A.1) and thus assertion (ii) of theorem 4.1 is proved.

\section{References}

[1] L. Amour, B. Grébert, and J.-C. Guillot. L'électron habillé non relativiste dans un champ magnétique. C. R. Math. Acad. Sci. Paris, 340(6):421-426, 2005 .

[2] L. Amour, B. Grébert, and J.-C. Guillot. The dressed mobile atoms and ions. preprint, ArXiv:math-ph/0507052, 2005.

[3] A. Arai. Perturbation of embedded eigenvalues: a general class of exactly soluble models in Fock spaces. Hokkaido Math. J., 19(1):1-34, 1990.

[4] A. Arai. A particle-field Hamiltonian in relativistic quantum electrodynamics. J. Math. Phys., 41(7):4271-4283, 2000.

[5] A. Arai and M. Hirokawa. On the existence and uniqueness of ground states of a generalized spin-boson model. J. Funct. Anal., 151(2):455-503, 1997.

[6] J. Avron, I. Herbst, and B. Simon. Schrödinger operators with magnetic fields II. Separation of the center of mass in homogeneous magnetic fields. Ann. Phys., 114:431-451, 1978.

[7] J. Avron, I. Herbst, and B. Simon. Schrödinger operators with magnetic fields I. General interactions. Duke Math. J., 48:847-883, 1978.

[8] V. Bach, J. Fröhlich, and I. Sigal. Quantum electrodynamics of confined relativistic particles. Adv. Math., 137:205-298, 1998. 
[9] V. Bach, J. Fröhlich, and I. Sigal. Renormalization group analysis of spectral problems in quantum field theory. Adv. Math., 137:299-395, 1998.

[10] V. Bach, J. Fröhlich, and I. Sigal. Spectral analysis for systems of atoms and molecules coupled to the quantized radiation field. Comm. Math. Phys., 207:249-290, 1999.

[11] J.-M. Barbaroux, T. Chen, and S. Vugalter. Binding conditions for atomic $N$-electron systems in non-relativistic QED. Ann. Henri Poincaré, 4(6):1101-1136, 2003.

[12] T. Chen. Operator-theoretic infrared renormalization and construction of dressed 1-particle states. preprint mp-arc 01-310, 2001.

[13] C. Cohen-Tannoudji. Introduction to quantum electrodynamics. In G. Grynberg and R. Stora, editors, Tendances actuelles en physique atomique / New trends in atomic physics. Elsevier Science, 1984.

[14] C. Cohen-Tannoudji, J. Dupont-Roc, and G. Grynberg. Processus d'interaction entre photons et atomes. Editions du CNRS, 2001.

[15] H. L. Cycon, R. G. Fröese, W. Kirsch, and B. Simon. Schrödinger operators with application to quantum mechanics and global geometry. Texts and Monographs in Physics. Springer-Verlag, Berlin, study edition, 1987.

[16] J. Dereziński and C. Gérard. Asymptotic completeness in quantum field theory. Massive Pauli-Fierz Hamiltonians. Rev. Math. Phys., 11:383-450, 1999.

[17] M. Dimassi and G. D. Raikov. Spectral asymptotics for quantum Hamiltonians in strong magnetic fields. preprint LAGA Université Paris Nord, 2004 .

[18] J. Fröhlich. On the infrared problem in a model of scalar electrons and massless, scalar bosons. Ann. Inst. H. Poincaré Sect. A (N.S.), 19:1-103, 1973.

[19] J. Fröhlich. On the infrared problem in a model of scalar electrons and massless scalar bosons. Fortschr. Phys., 22:159-198, 1974.

[20] J. Fröhlich, M. Griesemer, and B. Schlein. Asymptotic completeness for Rayleigh scattering. Ann. Inst. Henri Poincaré, 3:107-170, 2002.

[21] J. Fröhlich, M. Griesemer, and B. Schlein. Asymptotic completeness for Compton scattering. Comm. Math. Phys., 252:415-476, 2004.

[22] C. Gérard and I. Łaba. Multiparticle quantum scattering in constant magnetic fields, volume 90 of Mathematical Surveys and Monographs. American Mathematical Society, Providence, RI, 2002. 
[23] M. Griesemer. Non-relativistic matter and quantized radiation. preprint, ArXiv:math.ph/0410033, 2004.

[24] M. Griesemer, E. Lieb, and M. Loss. Ground states in non-relativistic quantum electrodynamics. Inv. Math., 145:557-595, 2001.

[25] F. Hiroshima. Ground states of a model in nonrelativistic quantum electrodynamics. I. J. Math. Phys., 40(12):6209-6222, 1999.

[26] F. Hiroshima. Ground states of a model in nonrelativistic quantum electrodynamics. II. J. Math. Phys., 41(2):661-674, 2000.

[27] F. Hiroshima. Ground states and spectrum of quantum electrodynamics of non-relativistic particles. Trans. Amer. Math. Soc., 353:4497-4528, 2001.

[28] F. Hiroshima. Analysis of ground states of atoms interacting with a quantized radiation field. In Topics in the theory of Schrödinger operators. World Scientific, 2004.

[29] F. Hiroshima. Multiplicity of ground states in quantum field models: application of asymptotic fields. preprint, ArXiv:math-ph/0402075, 2004.

[30] F. Hiroshima and K. R. Ito. Mass renormalization in non-relativistic quantum electrodynamics with spin 1/2. preprint, mp-arc 04-406, 2004.

[31] A. Itwatsuka and H. Tamura. Asymptotics distribution of eigenvalues for Pauli operators with non constant magnetic fields. Duke Math. J., 93:535574, 1998.

[32] E. Lieb and M. Loss. Existence of atoms and molecules in non-relativistic quantum electrodynamics. Adv. Theor. Math. Phys., 7:667-710, 2003.

[33] E. Lieb and M. Loss. A note on polarization vectors in quantum electrodynamics. Comm. Math. Phys., 252:477-483, 2004.

[34] J. S. Møller. The translation invariant massive Nelson model : The bottom of the spectrum. preprint, mp-arc 04-258, 2004.

[35] A. Pizzo. One particle (improper) states in Nelson's massless model. Ann. Henri Poincaré, 4:439-486, 2003.

[36] A. Pizzo. Scattering of an infraparticle : the one-particle sector in Nelson's massless model. preprint mp-arc 04-103, 2004.

[37] G. Raikov. Eigenvalue asymptotics for the Pauli operator in strong nonconstant magnetic fields. Ann. Inst. Fourier, 49:1603-1636, 1999.

[38] M. Reed and B. Simon. Methods of modern mathematical physics. II. Fourier analysis, self-adjointness. Academic Press [Harcourt Brace Jovanovich Publishers], New York, 1975. 
[39] I. Shigekawa. Spectral properties of Schrödinger operators with magnetic fields for a spin $\frac{1}{2}$ particle. J. Funct. Anal., 101(2):255-285, 1991.

[40] A. V. Sobolev. On the Lieb-Thirring estimates for the Pauli operator. Duke Math. J., 82(3):607-635, 1996.

[41] H. Spohn. Dynamics of charged particles and their radiation field. Cambridge university press, 2004. 